\title{
Entropy fluctuation theorems in driven open systems: Application to electron counting statistics
}

\author{
Massimiliano Esposito,* Upendra Harbola, and Shaul Mukamel \\ Department of Chemistry, University of California, Irvine, California 92697, USA \\ (Received 30 April 2007; revised manuscript received 4 July 2007; published 26 September 2007)
}

\begin{abstract}
The total entropy production generated by the dynamics of an externally driven systems exchanging energy and matter with multiple reservoirs and described by a master equation is expressed as the sum of three contributions, each corresponding to a distinct mechanism for bringing the system out of equilibrium: Nonequilibrium initial conditions, external driving, and breaking of detailed balance. We derive three integral fluctuation theorems (FTs) for these contributions and show that they lead to the following universal inequality: An arbitrary nonequilibrium transformation always produces a change in the total entropy production greater than or equal to the one produced if the transformation is done very slowly (adiabatically). Previously derived fluctuation theorems can be recovered as special cases. We show how these FTs can be experimentally tested by performing the counting statistics of the electrons crossing a single level quantum dot coupled to two reservoirs with externally varying chemical potentials. The entropy probability distributions are simulated for driving protocols ranging from the adiabatic to the sudden switching limit.
\end{abstract}

DOI: 10.1103/PhysRevE.76.031132

PACS number(s): 05.70.Ln, 05.40.-a, 73.63.-b

\section{INTRODUCTION}

In statistical mechanics, thermodynamic laws are recovered at the level of ensemble averages. The past decade has brought new insights into nonequilibrium statistical mechanics due to the discovery of various types of fluctuation relations valid arbitrarily far from equilibrium [1-17]. These relations identify, at the level of the single realization of a statistical ensemble, the "trajectory entropy" which upon ensemble averaging reproduces the thermodynamic entropy. They therefore quantify the statistical significance of nonthermodynamic behaviors which can become significant in small systems $[18,19]$. Various experimental verifications of these FTs have been reported [20-26].

In this paper, we consider an open system, described by a master equation (ME), exchanging matter and energy with multiple reservoirs. The system can be externally driven by varying its energies or the different temperature or chemical potentials of the reservoirs. There are three mechanisms for bringing such a system out of equilibrium: preparing it in a nonequilibrium state, externally driving it, or putting it in contact with multiple reservoirs at different temperatures or chemical potentials thus breaking the detailed balance condition (DBC). We show that each of these mechanisms make a distinct contribution to the total entropy production (EP) generated by the nonequilibrium dynamics of the system. The two first contributions are nonzero only if the system is not in its steady state and are therefore called nonadiabatic. The third contribution is equal to the EP for slow transformation during which the system remains in a steady-state and is therefore called adiabatic. We derive three FTs, for the total EP and its nonadiabatic and adiabatic contribution and show that they lead to exact inequalities valid arbitrary far from equilibrium. Previously derived FTs are recovered by

\footnotetext{
*Also at Center for Nonlinear Phenomena and Complex Systems, Universite Libre de Bruxelles, Code Postal 231, Campus Plaine, B-1050 Brussels, Belgium.
}

considering specific types of nonequilibrium transformations. Steady state FTs $[6,12,13]$ are obtained for systems maintained in a nonequilibrium steady state (NESS) between reservoirs with different thermodynamic properties. The Jarzynski or Crooks type FTs $[4,8,9]$ are derived for systems initially at equilibrium with a single reservoir which are externally driven out of equilibrium by an external force. The Hatano-Sasa FT $[10,11]$ is recovered for externally driven systems initially in a NESS with multiple reservoirs.

To calculate the statistical properties of the various contributions to the total EP and to demonstrate the FTs, we extend the generating function (GF) method [6] to driven open systems. Apart from providing clear proofs of the various FTs, this method is useful for simulations because it does not require to explicitly generate the stochastic trajectories. Some additional insight is provided by using an alternative derivation of the FTs similar to the Crooks derivation $[8,9]$, where the total EP and its nonadiabatic part can be identified in terms of forward-backward trajectory probabilities. By doing so, we connect the trajectory approach previously used for driven closed systems $[8,9,14]$ with the GF approach used for steady state systems $[6,12]$.

We propose to experimentally test these new FTs in a driven single orbital quantum dot where the various entropy probability distributions can be measured by the full electron counting statistics which keeps track of the four possible types of electron transfer (in and out of the dot through either lead). Such measurements of the bidirectional counting statistics have become feasible recently [27]. We calculate the entropy probability distributions, analyze their behavior as the driving is varied between the sudden and the adiabatic limits, and verify the validity of the FTs.

In Sec. II we present our stochastic model and in Sec. III we describe the various contributions to the total EP generated during a nonequilibrium transformation and the inequalities that these contributions satisfy. In Sec. IV, we define the various trajectory entropies which upon ensemble averaging give the various contributions to the EP. We then present the GF formalism used to calculate the statistical properties of these trajectory entropies. In Sec. V, we derive 
the various FTs and the implied inequalities. Alternative derivations of FTs in terms of forward-backward trajectories are given in the Appendix A. By considering specific nonequilibrium transformations, we recover most of the previously derived FTs. Finally in Sec. VI, we apply our results to the full counting statistics of electrons in a driven quantum dot. Conclusions are drawn in Sec. VII.

\section{MASTER EQUATION}

We consider an externally driven open system exchanging particles and energy with multiple reservoirs. Each state $m$ of the system has a given energy $\epsilon_{m}$ and $N_{m}$ particles. The total number of states $m$ is finite and equal to $M$. The probability to find the system in a state $m$ at time $t$ is denoted by $p_{m}(t)$. The evolution of this probability is described by the ME

$$
\dot{p}_{m}(t)=\sum_{m^{\prime}} W_{m, m^{\prime}}\left(\lambda_{t}\right) p_{m^{\prime}}(t),
$$

where the rate matrix satisfies

$$
\sum_{m} W_{m, m^{\prime}}\left(\lambda_{t}\right)=0
$$

We assume that if a transition from $m$ to $m^{\prime}$ can occur, the reversed transition from $m^{\prime}$ to $m$ can also occur. Various parameters, such as the energies $\epsilon_{m}$ of the system or the chemical potential $\mu_{\nu}$ and the temperature $\beta_{\nu}^{-1}$ of the $\nu$ reservoir can be varied in time externally according to a known protocol. This is described by the dependence of the rate matrix on several time-dependent parameters $\lambda_{t}$. If the transition rates are kept constant, the system will eventually reach the unique steady state solution $p_{m}^{\text {st }}(\lambda)$ which satisfies $\dot{p}_{m}^{\text {st }}(\lambda)=0[28]$.

The transition rates will be expressed as sums of contributions from different reservoirs $\nu$

$$
W_{m, m^{\prime}}(\lambda)=\sum_{\nu} W_{m, m^{\prime}}^{(\nu)}(\lambda),
$$

each satisfying

$$
\begin{aligned}
\frac{W_{m^{\prime}, m}^{(\nu)}(\lambda)}{W_{m, m^{\prime}}^{(\nu)}(\lambda)}= & \exp \left\{\beta _ { \nu } ( \lambda ) \left[\left(\epsilon_{m}(\lambda)-\epsilon_{m^{\prime}}(\lambda)\right)\right.\right. \\
& \left.\left.-\mu_{\nu}(\lambda)\left(N_{m}-N_{m^{\prime}}\right)\right]\right\} .
\end{aligned}
$$

If all reservoirs have the same thermodynamic properties (temperature $\beta^{-1}$ and chemical potential $\mu$ ), the steady state distribution coincides with the equilibrium distribution $p_{m}^{\text {st }}(\lambda)=p_{m}^{\text {eq }}(\lambda)$ which satisfies the detailed balanced condition (DBC)

$$
W_{m, m^{\prime}}^{(\nu)}(\lambda) p_{m^{\prime}}^{\mathrm{eq}}(\lambda)=W_{m^{\prime}, m}^{(\nu)}(\lambda) p_{m}^{\mathrm{eq}}(\lambda) .
$$

As a consequence of Eqs. (4) and (5), the equilibrium distribution then assumes the grand canonical form

$$
p_{m}^{\mathrm{eq}}(\lambda)=\frac{\exp \left[-\beta(\lambda)\left(\epsilon_{m}(\lambda)-\mu(\lambda) N_{m}\right)\right]}{\Xi(\lambda)},
$$

where $\Xi(\lambda)$ is the grand canonical partition function. However, in the general case where the reservoirs have different $\beta^{(\nu)}$ and $\mu^{(\nu)}$, the DBC does not hold and $p_{m}^{\text {st }}(\lambda)$ is a NESS.

\section{ENTROPIES}

The Gibbs entropy of the system is a state function defined as

$$
S(t) \equiv-\sum_{m} p_{m}(t) \ln p_{m}(t) .
$$

Using Eqs. (1) and (2), the system EP reads

$$
\dot{S}(t)=-\sum_{m} \dot{p}_{m}(t) \ln p_{m}(t)=-\sum_{m, m^{\prime}} W_{m, m^{\prime}}\left(\lambda_{t}\right) p_{m^{\prime}}(t) \ln \frac{p_{m}(t)}{p_{m^{\prime}}(t)} .
$$

This can be partitioned as [12,14,32-34]

$$
\dot{S}(t)=\dot{S}_{\mathrm{tot}}(t)-\dot{S}_{r}(t)
$$

with the total EP

$$
\dot{S}_{\text {tot }}(t) \equiv-\sum_{m, m^{\prime}, \nu} W_{m, m^{\prime}}^{(\nu)}\left(\lambda_{t}\right) p_{m^{\prime}}(t) \ln \frac{W_{m^{\prime}, m}^{(\nu)}\left(\lambda_{t}\right) p_{m}(t)}{W_{m, m^{\prime}}^{(\nu)}\left(\lambda_{t}\right) p_{m^{\prime}}(t)} \geqslant 0
$$

and the reservoir EP (also called medium entropy or entropy flow)

$$
\dot{S}_{r}(t) \equiv-\sum_{m, m^{\prime}, \nu} W_{m, m^{\prime}}^{(\nu)}\left(\lambda_{t}\right) p_{m^{\prime}}(t) \ln \frac{W_{m^{\prime}, m}^{(\nu)}\left(\lambda_{t}\right)}{W_{m, m^{\prime}}^{(\nu)}\left(\lambda_{t}\right)} .
$$

We note that $\dot{S}_{\text {tot }}(t) \geqslant 0$ follows from $W_{m, m^{\prime}}^{(\nu)}\left(\lambda_{t}\right) p_{m^{\prime}}(t)>0$ if $m^{\prime} \neq m$ and $\ln x \leqslant x-1$ for $x>0$ (if $m^{\prime}=m$ the log in zero), by using the fact that $\sum_{m, \nu} W_{m^{\prime}, m}^{(\nu)}\left(\lambda_{t}\right) p_{m}^{\text {st }}\left(\lambda_{t}\right)=0$ and $\sum_{m, \nu} W_{m, m^{\prime}}^{(\nu)}\left(\lambda_{t}\right)=0 . \dot{S}(t)$ is the contribution to $\dot{S}_{\text {tot }}(t)$ coming from the changes in the system probability distribution and $\dot{S}_{r}(t)$ is the contribution coming from matter and energy exchange processes between the system and its reservoirs.

We further separate the reservoir EP into two components $[11,15]$

$$
\dot{S}_{r}(t) \equiv \dot{S}_{\mathrm{ex}}(t)+\dot{S}_{a}(t)
$$

with the excess $E P$

$$
\dot{S}_{\mathrm{ex}}(t) \equiv-\sum_{m, m^{\prime}} W_{m, m^{\prime}}\left(\lambda_{t}\right) p_{m^{\prime}}(t) \ln \frac{p_{m^{\prime}}^{\mathrm{st}}\left(\lambda_{t}\right)}{p_{m}^{\mathrm{st}}\left(\lambda_{t}\right)}=\sum_{m} \dot{p}_{m}(t) \ln p_{m}^{\mathrm{st}}\left(\lambda_{t}\right)
$$

and the adiabatic EP (also called housekeeping entropy $[11,15])$ 


$$
\begin{aligned}
\dot{S}_{a}(t) \equiv & -\sum_{m, m^{\prime}, \nu} W_{m, m^{\prime}}^{(\nu)}\left(\lambda_{t}\right) p_{m^{\prime}}(t) \\
& \times \ln \frac{W_{m^{\prime}, m}^{(\nu)}\left(\lambda_{t}\right) p_{m}^{\mathrm{st}}\left(\lambda_{t}\right)}{W_{m, m^{\prime}}^{(\nu)}\left(\lambda_{t}\right) p_{m^{\prime}}^{\mathrm{st}}\left(\lambda_{t}\right)} \geqslant 0 .
\end{aligned}
$$

The positivity of Eq. (14) follows from the same reason as Eq. (10). If a transformation is done very slowly, the system remains at all times in the steady state distribution $p_{m}(t)$ $=p_{m}^{\text {st }}\left(\lambda_{t}\right)$. Such a transformation is called adiabatic. We then have $\dot{S}_{\text {tot }}(t)=\dot{S}_{a}(t)$ and $\dot{S}_{\text {ex }}(t)=-\dot{S}(t)$. We also notice that $\dot{S}_{a}(t)=0$ when the DBC is satisfied.

We next define the state function quantity

$$
S_{b}(t) \equiv-\sum_{m} p_{m}(t) \ln \frac{p_{m}(t)}{p_{m}^{\text {st }}\left(\lambda_{t}\right)}
$$

which is obviously zero when the system is at steady state. When considering a transformation between steady states, $\Delta S_{b}(T, 0)=\int_{0}^{T} d \tau \dot{S}_{b}(\tau)=S_{b}(T)-S_{b}(0)=0$. We call $\dot{S}_{b}(t)$ the boundary EP (the terminology will be explained shortly) and separate it in two parts

$$
\dot{S}_{b}(t)=\dot{S}_{\mathrm{na}}(t)-\dot{S}_{d}(t),
$$

where the nonadiabatic EP is

$$
\begin{aligned}
\dot{S}_{\text {na }}(t) & \equiv-\sum_{m} \dot{p}_{m}(t) \ln \frac{p_{m}(t)}{p_{m}^{\text {st }}\left(\lambda_{t}\right)} \\
& =-\sum_{m, m^{\prime}} W_{m, m^{\prime}}\left(\lambda_{t}\right) p_{m^{\prime}}(t) \ln \frac{p_{m}(t) p_{m^{\prime}}^{\text {st }}\left(\lambda_{t}\right)}{p_{m}^{\text {st }}\left(\lambda_{t}\right) p_{m^{\prime}}(t)} \geqslant 0,
\end{aligned}
$$

and the driving $E P$ is

$$
\dot{S}_{d}(t) \equiv \sum_{m} p_{m}(t) \dot{\phi}_{m}\left(\lambda_{t}\right)
$$

with

$$
\phi_{m}\left(\lambda_{t}\right) \equiv-\ln p_{m}^{\text {st }}\left(\lambda_{t}\right) .
$$

The positivity of Eq. (17) is again shown in the same way as for Eqs. (10) and (14). If no external driving acts on the system, $\lambda$ is time independent and from Eq. (18), $\dot{S}_{d}(t)=0$. For an adiabatic transformation, since $p_{m}(t)=p_{m}^{\text {st }}\left(\lambda_{t}\right)$, from Eqs. (15) and (17), we see that $\dot{S}_{\text {na }}(t)=0$ as well as $\dot{S}_{b}(t)=0$. From Eq. (16), this also means that $\dot{S}_{d}(t)=0$. Therefore, $\dot{S}_{d}(t) \neq 0$ only for nonadiabatic driving. Using Eq. (17) with Eqs. (8) and (13), we find

$$
\dot{S}_{\text {na }}(t)=\dot{S}_{\text {ex }}(t)+\dot{S}(t)=\dot{S}_{\text {tot }}(t)-\dot{S}_{a}(t) .
$$

It is clear from the last equality why we call $\dot{S}_{\text {na }}(t)$ the nonadiabatic EP. The inequality $\dot{S}_{\text {ex }}(t) \geqslant-\dot{S}(t)$ which follows from the first line is a generalization of the "second law of steady state thermodynamics" $[11,35,36]$ derived for transitions between steady states.

We next summarize our results

$$
\begin{gathered}
\dot{S}_{\mathrm{tot}}(t)=\dot{S}_{\mathrm{na}}(t)+\dot{S}_{a}(t) \geqslant 0, \\
\dot{S}_{\mathrm{na}}(t)=\dot{S}_{d}(t)+\dot{S}_{b}(t) \geqslant 0, \\
\dot{S}_{a}(t) \geqslant 0 .
\end{gathered}
$$

The total EP is always positive and can be separated into two positive contributions, adiabatic (which are nonzero only when the DBC is violated) and nonadiabatic effects. The latter can be due to a nonadiabatic external driving acting on the system or to the fact that one considers transformation during which the system is initially or finally not in a steady state. We therefore have a minimum EP principle stating that the total EP for arbitrary nonequilibrium transformations takes its minimal value if the transformation is done adiabatically (very slowly). The equality sign in Eq. (21) is satisfied for adiabatic transformations which occur at equilibrium. The equality sign in Eq. (22) holds for adiabatic transformations. The equality sign in Eq. (23) only occurs when the DBC is satisfied.

\section{TRAJECTORY ENTROPIES}

The evolution described by the ME can be represented by an ensemble of stochastic trajectories involving instantaneous jumps between states. This will allow us to define fluctuating (trajectory) entropies.

\section{A. Definitions}

We denote a trajectory taken by the system between $t=0$ and $t=T$ by

$$
m_{(\tau)}=\left\{0-m_{0} \stackrel{\tau_{1}}{\rightarrow} m_{1} \rightarrow \cdots m_{j-1} \stackrel{\tau_{j}}{\rightarrow} \stackrel{\tau_{j+1}}{\rightarrow} m_{j} \stackrel{\tau_{N}}{\rightarrow} \cdots m_{N-1} \rightarrow m_{N}-T\right\} .
$$

At $t=0$ the system is in $m_{0}$, and stays there until it jumps at $\tau_{1}$ to $m_{1}$, etc., jumps at $\tau_{N}$ from $m_{N-1}$ to $m_{N}$ and stays in $m_{N}$ until $t=T$ (see Fig. 1). $N$ is the total number of jumps during this trajectory.

We next introduce various types of "trajectory entropy production" (TEP). We will see at the end of this section that when ensemble averaged, these correspond to the various EP defined in Sec. III.

The trajectory Gibbs entropy is defined as

$$
s\left[m_{(\tau)}, t\right] \equiv-\ln p_{m_{(\tau)}}(t),
$$

where $p_{m_{(\tau)}}(t)$ represents the value of $p_{m}(t)$ along the trajectory $m_{(\tau)}$. The system TEP is given by

$$
\dot{s}\left[m_{(\tau)}, t\right]=-\left.\frac{\dot{p}_{m}(t)}{p_{m}(t)}\right|_{m_{(\tau)}}+\sum_{j=1}^{N} \delta\left(t-\tau_{j}\right) \ln \frac{p_{m_{j-1}}\left(\tau_{j}\right)}{p_{m_{j}}\left(\tau_{j}\right)} .
$$

The first term represents the smooth changes of $s\left[m_{(\tau)}, t\right]$ along the horizontal segments of the trajectory in Fig. 1 during which the system is in a well defined state. These changes are only due to the time dependence of the probability to be on a given state. The notation $\left.\right|_{m_{(\tau)}}$ means that the $m$ 




FIG. 1. Representation of a trajectory $m_{(\tau)}$.

in the expression changes depending on which horizontal segment along the trajectory one considers. The second term represents the discrete changes of $s\left[m_{(\tau)}, t\right]$ along the vertical segments of the trajectory. These changes are singular and only due to the change in the system state.

Separating the trajectory system TEP similarly as the system EP in Sec. III, we get

$$
\dot{s}_{\text {tot }}\left[m_{(\tau)}, t\right]=\dot{s}\left[m_{(\tau)}, t\right]+\dot{s}_{r}\left[m_{(\tau)}, t\right],
$$

where the total TEP is

$$
\begin{aligned}
\dot{s}_{\mathrm{tot}}\left[m_{(\tau)}, t\right] \equiv & -\left.\frac{\dot{p}_{m}(t)}{p_{m}(t)}\right|_{m_{(\tau)}}+\sum_{j=1}^{N} \delta\left(t-\tau_{j}\right) \\
& \times \ln \frac{W_{m_{j}, m_{j-1}}^{\left(\nu_{j}\right)}\left(\lambda_{\tau_{j}}\right) p_{m_{j-1}}\left(\tau_{j}\right)}{W_{m_{j-1}, m_{j}}^{\left(\nu_{j}\right)}\left(\lambda_{\tau_{j}}\right) p_{m_{j}}\left(\tau_{j}\right)},
\end{aligned}
$$

and the reservoir TEP

$$
\dot{s}_{r}\left[m_{(\tau)}, t\right] \equiv \sum_{j=1}^{N} \delta\left(t-\tau_{j}\right) \ln \frac{W_{m_{j} m_{j-1}}^{\left(\nu_{j}\right)}\left(\lambda_{\tau_{j}}\right)}{W_{m_{j-1}, m_{j}}^{\left(\nu_{j}\right)}\left(\lambda_{\tau_{j}}\right)} .
$$

We further separate the reservoir TEP into

$$
\dot{s}_{r}\left[m_{(\tau)}, t\right]=\dot{s}_{a}\left[m_{(\tau)}, t\right]+\dot{s}_{\mathrm{ex}}\left[m_{(\tau)}, t\right],
$$

with the adiabatic TEP

$$
\dot{s}_{a}\left[m_{(\tau)}, t\right] \equiv \sum_{j=1}^{N} \delta\left(t-\tau_{j}\right) \ln \frac{W_{m_{j}, m_{j-1}}^{\left(\nu_{j}\right)}\left(\lambda_{\tau_{j}}\right) p_{m_{j-1}}^{\mathrm{st}}\left(\lambda_{\tau_{j}}\right)}{W_{m_{j-1}, m_{j}}^{\left(\nu_{j}\right)}\left(\lambda_{\tau_{j}}\right) p_{m_{j}}^{\mathrm{st}}\left(\lambda_{\tau_{j}}\right)},
$$

and the excess TEP

$$
\dot{s}_{\mathrm{ex}}\left[m_{(\tau)}, t\right] \equiv \sum_{j=1}^{N} \delta\left(t-\tau_{j}\right) \ln \frac{p_{m_{j}}^{\mathrm{st}}\left(\lambda_{\tau_{j}}\right)}{p_{m_{j-1}}^{\mathrm{st}}\left(\lambda_{\tau_{j}}\right)} .
$$

The nonadiabatic TEP

$$
\dot{s}_{\mathrm{na}}\left[m_{(\tau)}, t\right] \equiv-\left.\frac{\dot{p}_{m}(t)}{p_{m}(t)}\right|_{m_{(\tau)}}+\sum_{j=1}^{N} \delta\left(t-\tau_{j}\right) \ln \frac{p_{m_{j-1}}\left(\tau_{j}\right) p_{m_{j}}^{\mathrm{st}}\left(\lambda_{\tau_{j}}\right)}{p_{m_{j-1}}^{\mathrm{st}}\left(\lambda_{\tau_{j}}\right) p_{m_{j}}\left(\tau_{j}\right)}
$$

is made of the sum of two terms

$$
\dot{s}_{\text {na }}\left[m_{(\tau)}, t\right] \equiv \dot{s}_{d}\left[m_{(\tau)}, t\right]+\dot{s}_{b}\left[m_{(\tau)}, t\right],
$$

the boundary TEP

$$
\begin{aligned}
\dot{s}_{b}\left[m_{(\tau)}, t\right] \equiv & -\left.\frac{\dot{p}_{m}(t)}{p_{m}(t)}\right|_{m_{(\tau)}}-\dot{\lambda_{t}} \frac{\partial \phi_{m_{(\tau)}}\left(\lambda_{t}\right)}{\partial \lambda_{t}} \\
& +\sum_{j=1}^{N} \delta\left(t-\tau_{j}\right) \ln \frac{p_{m_{j-1}}\left(\tau_{j}\right) p_{m_{j}}^{\mathrm{st}}\left(\lambda_{\tau_{j}}\right)}{p_{m_{j-1}}^{\mathrm{st}}\left(\lambda_{\tau_{j}}\right) p_{m_{j}}\left(\tau_{j}\right)},
\end{aligned}
$$

and the driving TEP

$$
\dot{s}_{d}\left[m_{(\tau)}, t\right] \equiv \dot{\lambda_{t}} \frac{\partial \phi_{m_{(\tau)}}\left(\lambda_{t}\right)}{\partial \lambda_{t}}
$$

As in Sec. III, since

$$
\dot{s}_{\text {na }}\left[m_{(\tau)}, t\right]=\dot{s}\left[m_{(\tau)}, t\right]+\dot{s}_{\text {ex }}\left[m_{(\tau)}, t\right],
$$

we get

$$
\dot{s}_{\text {tot }}\left[m_{(\tau)}, t\right]=\dot{s}_{\text {na }}\left[m_{(\tau)}, t\right]+\dot{s}_{a}\left[m_{(\tau)}, t\right] .
$$

We generically denote these TEP by $a\left[m_{(\tau)}, t\right]$. The change of $a\left[m_{(\tau)}, t\right]$ along a trajectory $m_{(\tau)}$ of length $T$ is given by

$$
\Delta a\left[m_{(\tau)}, T\right]=\int_{0}^{T} d t \dot{a}\left[m_{(\tau)}, t\right] .
$$

Notice that $\Delta s\left[m_{(\tau)}, T\right]$ and $\Delta s_{b}\left[m_{(\tau)}, T\right]$ are state function TEP 


$$
\Delta s\left[m_{(\tau)}, T\right]=\ln \frac{p_{m_{0}}(0)}{p_{m_{N}}(T)}=s_{m_{N}}(T)-s_{m_{0}}(0),
$$

where $s_{m}(t) \equiv-\ln p_{m}(t)$, and

$$
\Delta s_{b}\left[m_{(\tau)}, T\right]=\left[s_{m_{N}}(T)-\phi_{m_{N}}\left(\lambda_{T}\right)\right]-\left[s_{m_{0}}(0)-\phi_{m_{0}}\left(\lambda_{0}\right)\right] .
$$

The other TEP are path functions.

\section{B. Deriving trajectory entropies from measured currents}

Using Eq. (4), the reservoir TEP can be expressed as

$$
\dot{s}_{r}\left[m_{(\tau)}, t\right]=-\sum_{\nu} \beta_{\nu}\left(\lambda_{t}\right)\left(I_{\text {heat }}^{(\nu)}\left[m_{(\tau)}, t\right]-\mu_{\nu}\left(\lambda_{t}\right) I_{\text {mat }}^{(\nu)}\left[m_{(\tau)}, t\right]\right),
$$

where the heat current between the $\nu$ reservoir and the system is

$$
I_{\text {heat }}^{(\nu)}\left[m_{(\tau)}, t\right]=\sum_{j=1}^{N} \delta_{\nu, \nu_{j}}\left(t-\tau_{j}\right)\left[\epsilon_{m_{j}}\left(\lambda_{\tau_{j}}\right)-\epsilon_{m_{j-1}}\left(\lambda_{\tau_{j}}\right)\right]
$$

and the matter current between the $\nu$ reservoir and the system

$$
I_{\text {mat }}^{(\nu)}\left[m_{(\tau)}, t\right]=\sum_{j=1}^{N} \delta_{\nu, \nu_{j}}\left(t-\tau_{j}\right)\left(N_{m_{j}}-N_{m_{j-1}}\right) .
$$

The currents are positive if the system energy (matter) increases. $\delta_{\nu, \nu_{j}}\left(t-\tau_{j}\right)$ is a Dirac distribution centered at time $\tau_{j}$ only if the transition is due to the reservoir $\nu=\nu_{i}$. Otherwise, it is zero. We thus confirm that the reservoir EP is the entropy associated to system-reservoir exchange processes.

We assume that the parametric time dependence of the energies, temperatures, and chemical potentials is known. Except in degenerate cases for which two different transitions between states have the same energy difference and number of particle difference, the trajectory of the system can be uniquely determined by measuring the heat and matter currents between the system and the reservoirs. The system steady state probability distribution can be calculated by recording the steady state currents for sufficiently long times for different values of the energies, temperatures, or chemical potentials. The driven system probability distribution can in principle be calculated by reproducing the measurement of the currents multiple times. All trajectory entropies containing the logarithm of the transition rates can be expressed in terms of a combination of the reservoir EP (directly measurable via current) and other trajectory entropies which can be expressed in terms of the system probability distribution (actual or steady state). Therefore, provided the current measurements can be repeated often enough to get good statistics, all the trajectory entropies are in principle measurable.

\section{Statistical properties using generating functions}

The GF formalism allows to compute the probability distributions and all statistical properties of the TEP without having to generate the trajectories themselves. It further provides a direct means for proving the FTs.

The GF associated with the changes of $a\left[m_{(\tau)}, t\right]$ along a trajectory is given by

$$
G(\gamma, t) \equiv\left\langle\exp \left\{\gamma \Delta a\left[m_{(\tau)}, t\right]\right\}\right\rangle,
$$

where $\langle\cdot\rangle$ denotes an average over all possible trajectories. The probability that the system follows a trajectory with the constraint $\Delta A=\Delta a\left[m_{(\tau)}, t\right]$ at time $t$ can be obtained from the GF using

$$
P(\Delta A, t) \equiv\left\langle\delta\left(\Delta A-\Delta a\left[m_{(\tau)}, t\right]\right)\right\rangle=\frac{1}{2 \pi} \int_{-\infty}^{\infty} d \gamma e^{-i \gamma \Delta A} G(i \gamma, t) .
$$

By inverting Eq. (44), we get

$$
G(i \gamma, t)=\int_{-\infty}^{\infty} d \Delta A e^{i \gamma \Delta A} P(\Delta A, t) .
$$

The moments of the distribution are given by derivatives of the GF

$$
\left\langle\Delta a^{k}\left[m_{(\tau)}, t\right]\right\rangle=\left.\frac{\partial^{k} G(\gamma, t)}{\partial \gamma^{k}}\right|_{\gamma=0}, \quad k=1,2, \ldots .
$$

In order to compute the GF, we recast it in the form

$$
G(\gamma, t)=\sum_{m} g_{m}(\gamma, t)
$$

where

$$
g_{m}(\gamma, t)=p_{m}(t)\left\langle\exp \left\{\gamma \Delta a\left[m_{(\tau)}, t\right]\right\}\right\rangle_{m}
$$

is the product of the probability to find the system in state $m$ at time $t$ multiplied by the expectation value of $\exp \left\{\gamma \Delta a\left[m_{(\tau)}, t\right]\right\}$ conditional on the system being in state $m$ at time $t$. Since $\Delta a\left[m_{(\tau)}, t\right]=0$ for a trajectory of length $t=0$, we have $g_{m}(\gamma, 0)=p_{m}(0)$. We also have $G(0, t)=1$ and $g_{m}(0, t)=p_{m}(t)$.

The time derivative of Eq. (47) gives

$$
\dot{G}(\gamma, t)=\sum_{m} \dot{g}_{m}(\gamma, t),
$$

where $\dot{g}_{m}(\gamma, t)$ depends on the TEP of interest. Below, we will derive equations of motion for the $g_{m}(\gamma, t)$ 's associated to the various TEP.

\section{State function trajectory entropy production}

The generating function associated with a state function TEP $\Delta a\left[m_{(\tau)}, t\right]=a_{m}(t)-a_{n}(0)$ like $\Delta s\left[m_{(\tau)}, t\right]$ or $\Delta s_{b}\left[m_{(\tau)}, t\right]$ may be straightforwardly obtained using Eq. (47) with Eq. (48). We get

$$
G(\gamma, t)=\sum_{m, n} \exp \left\{\gamma\left[a_{m}(t)-a_{n}(0)\right]\right\} p_{m}(t) p_{n}(0) .
$$

\section{Excess trajectory entropy production}

$s_{\mathrm{ex}}\left[m_{(\tau)}, t\right]$ acquires an amount $s_{\mathrm{ex}}\left(m, m^{\prime}\right)=\ln \left[p_{m}^{\mathrm{st}}\left(\lambda_{t}\right) /\right.$ $\left.p_{m^{\prime}}^{\text {st }}\left(\lambda_{t}\right)\right]$ each time a transition from a state $m^{\prime}$ to $m$ occurs 
and it remains constant along a given state $m$ of the system. This means that

$$
\begin{aligned}
\dot{g}_{m}^{(\mathrm{ex})}(\gamma, t)= & \sum_{m^{\prime}} W_{m, m^{\prime}}\left(\lambda_{t}\right) p_{m^{\prime}}(t)+\exp \left\{\gamma s_{\mathrm{ex}}\left(m, m^{\prime}\right)\right\} \\
& \times\left\langle\exp \left\{\gamma \Delta s_{\mathrm{ex}}\left[m_{(\tau)}, t\right]\right\}\right\rangle_{m^{\prime}},
\end{aligned}
$$

which using Eq. (48) can be rewritten

$$
\dot{g}_{m}^{(\mathrm{ex})}(\gamma, t)=\sum_{m^{\prime}}\left(\frac{p_{m}^{\mathrm{st}}\left(\lambda_{t}\right)}{p_{m^{\prime}}^{\mathrm{st}}\left(\lambda_{t}\right)}\right)^{\gamma} W_{m, m^{\prime}}\left(\lambda_{t}\right) g_{m^{\prime}}^{(\mathrm{ex})}(\gamma, t) .
$$

\section{Reservoir trajectory entropy production and currents}

Each time a transition along the system trajectory occurs, $s_{r}\left[m_{(\tau)}, t\right]$ acquires an amount $s_{r}\left(m, m^{\prime}\right)=\ln \left(W_{m, m^{\prime}}^{(\nu)}\left(\lambda_{t}\right) /\right.$ $\left.W_{m^{\prime}, m}^{(\nu)}\left(\lambda_{t}\right)\right)$. Similarly to the excess entropy, we get

$$
\dot{g}_{m}^{(r)}(\gamma, t)=\sum_{\nu, m^{\prime}}\left(\frac{W_{m, m^{\prime}}^{(\nu)}\left(\lambda_{t}\right)}{W_{m^{\prime}, m}^{(\nu)}\left(\lambda_{t}\right)}\right)^{\gamma} W_{m, m^{\prime}}^{(\nu)}\left(\lambda_{t}\right) g_{m^{\prime}}^{(r)}(\gamma, t) .
$$

This equation has been used in the study of steady state FTs $[6,12,37]$.

In Eq. (40), we expressed the reservoir TEP in terms of currents. The time integrated individual currents give the heat and matter transfer between the $\nu$ reservoir and the system $q_{\text {heat }}^{(\nu)}\left[m_{(\tau)}, t\right]=\int_{0}^{t} d t^{\prime} I_{\text {heat }}^{(\nu)}\left[m_{(\tau)}, t^{\prime}\right]$ and $q_{\text {mat }}^{(\nu)}\left[m_{(\tau)}, t\right]$ $=\int_{0}^{t} d t^{\prime} I_{\text {mat }}^{(\nu)}\left[m_{(\tau)}, t^{\prime}\right]$. Their statistics can be calculated using

$$
\begin{aligned}
\dot{g}_{m}(\vec{\gamma}, t)= & \sum_{\nu, m^{\prime}} \exp \left\{\gamma_{\text {heat }}^{(\nu)}\left[\epsilon_{m}\left(\lambda_{t}\right)-\epsilon_{m^{\prime}}\left(\lambda_{t}\right)\right]\right\} \\
& \times \exp \left\{\gamma_{\text {mat }}^{(\nu)}\left(N_{m}-N_{m^{\prime}}\right)\right\} W_{m, m^{\prime}}^{(\nu)}\left(\lambda_{t}\right) g_{m^{\prime}}(\vec{\gamma}, t),
\end{aligned}
$$

where $\vec{\gamma}$ is a vector whose elements are the different $\gamma_{\text {heat }}^{(\nu)}$ s and $\gamma_{\text {mat }}^{(\nu)}$ 's. The GF calculated from Eq. (54) is therefore associated with the joint probability distribution for having a certain heat and matter transfer with each reservoir.

\section{Adiabatic trajectory entropy production}

Each time a transition along the system trajectory occurs, $s_{a}\left[m_{(\tau)}, t\right]$ acquires an amount $s_{a}\left(m, m^{\prime}\right)$ $=\ln \left[W_{m, m^{\prime}}^{(\nu)}\left(\lambda_{t}\right) p_{m^{\prime}}^{\mathrm{st}}\left(\lambda_{t}\right) / W_{m^{\prime}, m}^{(\nu)}\left(\lambda_{t}\right) p_{m}^{\mathrm{st}}\left(\lambda_{t}\right)\right]$. We therefore get

$$
\dot{g}_{m}^{(a)}(\gamma, t)=\sum_{\nu, m^{\prime}}\left(\frac{W_{m, m^{\prime}}^{(\nu)}\left(\lambda_{t}\right) p_{m^{\prime}}^{\mathrm{st}}\left(\lambda_{t}\right)}{W_{m^{\prime}, m}^{(\nu)}\left(\lambda_{t}\right) p_{m}^{\mathrm{st}}\left(\lambda_{t}\right)}\right)^{\gamma} W_{m, m^{\prime}}^{(\nu)}\left(\lambda_{t}\right) g_{m^{\prime}}^{(a)}(\gamma, t) .
$$

\section{Total trajectory entropy production}

Each time a transition along the system trajectory occurs, $s_{\text {tot }}\left[m_{(\tau)}, t\right]$ acquires an amount $\ln \left[W_{m, m^{\prime}}^{(\nu)}\left(\lambda_{t}\right) p_{m^{\prime}}(t) /\right.$ $\left.W_{m^{\prime}, m}^{(\nu)}\left(\lambda_{t}\right) p_{m}(t)\right]$. In addition it also changes by an amount $-d\left[\ln p_{m}(t)\right] / d t$ during an infinitesimally small time on a given state $m$ of the system. Combining the two, we have

$$
\begin{aligned}
\dot{g}_{m}^{(\mathrm{tot})}(\gamma, t)= & -\gamma\left(\frac{\dot{p}_{m}(t)}{p_{m}(t)}\right) g_{m}^{(\mathrm{tot})}(\gamma, t) \\
& +\sum_{\nu, m^{\prime}}\left(\frac{W_{m, m^{\prime}}^{(\nu)}\left(\lambda_{t}\right) p_{m^{\prime}}(t)}{W_{m^{\prime}, m}^{(\nu)}\left(\lambda_{t}\right) p_{m}(t)}\right)^{\gamma} W_{m, m^{\prime}}^{(\nu)}\left(\lambda_{t}\right) g_{m^{\prime}}^{(\mathrm{tot})}(\gamma, t) .
\end{aligned}
$$

\section{Nonadiabatic trajectory entropy production}

Like the total TEP, $s_{\text {na }}\left[m_{(\tau)}, t\right]$ acquires an amount $\ln \left[p_{m}^{\text {st }}\left(\lambda_{t}\right) p_{m^{\prime}}(t) / p_{m^{\prime}}^{\text {st }}\left(\lambda_{t}\right) p_{m}(t)\right]$ each time a transition from a states $m^{\prime}$ to $m$ occurs, and also changes by an amount $-d\left[\ln p_{m}(t)\right] / d t$ during an infinitesimally small time on a given state $m$. This gives

$$
\begin{aligned}
\dot{g}_{m}^{(\text {na) }}(\gamma, t)= & -\gamma\left(\frac{\dot{p}_{m}(t)}{p_{m}(t)}\right) g_{m}^{\text {(na) }}(\gamma, t) \\
& +\sum_{m^{\prime}}\left(\frac{p_{m}^{\text {st }}\left(\lambda_{t}\right) p_{m^{\prime}}(t)}{p_{m^{\prime}}^{\text {st }}\left(\lambda_{t}\right) p_{m}(t)}\right)^{\gamma} W_{m, m^{\prime}}\left(\lambda_{t}\right) g_{m^{\prime}}^{(\text {na })}(\gamma, t) .
\end{aligned}
$$

\section{Driving trajectory entropy production}

Since $\Delta s_{d}\left[m_{(\tau)}, t\right]$ exclusively accumulates along the segments of the system trajectory, we get

$\dot{g}_{m}^{(d)}(\gamma, t)=\gamma \dot{\phi}_{m}\left(\lambda_{t}\right) g_{m}^{(d)}(\gamma, t)+\sum_{m^{\prime}} W_{m, m^{\prime}}\left(\lambda_{t}\right) g_{m^{\prime}}^{(d)}(\gamma, t)$.

It follows from Eq. (46) that the average change of a TEP is obtained from its GF by differentiation with respect to $\gamma$ at $\gamma=0$. By differentiating the GF evolution equations of this section one recovers the evolution equation for the EPs of Sec. III. The EPs are therefore the ensemble average of the TEPs introduced in this section $\dot{A}(t)=\left\langle\dot{a}\left[m_{(\tau)}, t\right]\right\rangle$ and $\Delta A(T, 0)=\left\langle\Delta a\left[m_{(\tau)}, T\right]\right\rangle$.

\section{FLUCTUATION THEOREMS}

\section{A. General integral fluctuation theorems}

We can easily verify that $g_{m}(\gamma=-1, t)=p_{m}(t)$ is the solution of the evolution equations (56) and (57). It immediately follows from probability conservation and Eq. (49) that $\dot{G}_{\text {tot }}(-1, t)=\dot{G}_{\text {na }}(-1, t)=0$. Summing both sides of Eq. (55) over $m$, we also verify that $\dot{G}_{a}(-1, t)=0$. Because $g_{m}^{(z)}(-1,0)=p_{m}(0), G_{z}(-1,0)=1$ where $z=$ tot, na, $a$. Therefore, we find that $G_{z}(-1, t)=1$. Using Eq. (43), this results in the three FTs

$$
\begin{aligned}
& \left\langle\exp \left\{-\Delta s_{\text {tot }}\left[m_{(\tau)}, t\right]\right\}\right\rangle=1, \\
& \left\langle\exp \left\{-\Delta s_{\text {na }}\left[m_{(\tau)}, t\right]\right\}\right\rangle=1, \\
& \left\langle\exp \left\{-\Delta s_{a}\left[m_{(\tau)}, t\right]\right\}\right\rangle=1 .
\end{aligned}
$$

These FTs hold irrespective of the initial condition and the type of driving. Using Jensen's inequality $\left\langle e^{x}\right\rangle \geqslant e^{\langle x\rangle}$, they imply the inequalities (21)-(23). 
Equation (59) is the generalization to open systems of the integral FT for the TEP obtained earlier for closed systems [14]. The TEP (27) needs to specify which reservoir is responsible for the transitions occurring along the trajectory (by labeling the rates with reservoir index). This point, made earlier for an open system at steady state [12], is generalized here for driven systems with an arbitrary initial condition. Equation (60) will be shown in the next section to reduce to the integral Hatano-Sasa FT [11] for systems initially in a steady state. Equation (61) generalizes the integral FT for the adiabatic entropy [15] previously derived for closed system initially in a steady state.

Some additional insights can be gained by an alternative proof of the FTs (59) and (60) which use a forward-backward trajectory picture of the dynamics. This is given for completeness in the Appendix.

\section{B. Transitions between steady states}

We consider a system initially $(t=0)$ at steady state and subjected to an external driving force between $t=t_{\mathrm{di}}$ and $t$ $=t_{\mathrm{df}}$. For $t_{\mathrm{di}}>t>0$, the system remains in the steady state corresponding to $\lambda=\lambda_{t_{\mathrm{di}}}$. The time protocol of $\lambda_{t}$ during the driving $t_{\mathrm{df}}>t>t_{\mathrm{di}}$ is arbitrary. If $t_{\mathrm{tr}}$ is the characteristic transient time needed for the system to reach a steady state from an arbitrary distribution, for $t>t_{\mathrm{df}}+t_{\mathrm{tr}}$, the system is in the new steady state corresponding to $\lambda_{t_{\mathrm{df}}}$. The system is measured between $t=0$ and $t=T$.

\section{Fluctuation theorem for the reservoir entropy production}

We restrict our analysis to cases where the system is at steady state at $t=0$ and the driving starts at least a time $t_{\text {tr }}$ after the measurement started: $t_{\mathrm{di}}>t_{\mathrm{tr}}$.

We use the bra-ket notation where $|p(t)\rangle$ is the probability vector with components $p_{m}(t)$ and $\hat{\mathcal{W}}_{t}$ denotes the rate matrix. $|I\rangle$ denotes a vector with all components equal to one. The ME (1) now reads

$$
|\dot{p}(t)\rangle=\hat{\mathcal{W}}_{t}|p(t)\rangle .
$$

The generating function for the reservoir EP (53) for $\gamma=-1$ evolves according to the adjoint equation of Eq. (62)

$$
\left|\dot{g}^{(r)}(-1, t)\right\rangle=\hat{\mathcal{W}}_{t}^{\dagger}\left|g^{(r)}(-1, t)\right\rangle .
$$

The initial condition of Eqs. (62) and (63) is $|p(0)\rangle$ $=\left|g^{(r)}(-1,0)\right\rangle=\left|p^{\mathrm{st}}\left(\lambda_{t_{\mathrm{di}}}\right)\right\rangle$. The formal solution of Eq. (63) for $t<t_{\mathrm{di}}$ before the driving starts reads

$$
G_{r}(-1, t)=\left\langle I\left|\exp \left\{\hat{\mathcal{W}}^{\dagger} t\right\}\right| p_{m}^{\text {st }}\right\rangle .
$$

We now insert a closure relation in terms of right and left eigenvectors of the adjoint rate matrix between the evolution operator and the initial condition. Because the rate matrix and its adjoint have the same eigenvalues (all negative and one zero), for $t_{\mathrm{tr}}<t<t_{\mathrm{di}}$, only the right and left eigenvector associated with the zero eigenvalue survive. Since the right (left) eigenvector of $\hat{\mathcal{W}}^{\dagger}$ is $|I\rangle\left[\left\langle p_{m}^{\mathrm{st}}\left(\lambda_{t_{\mathrm{di}}}\right)\right|\right]$, we get for $t_{\mathrm{tr}}<t$ $<t_{\mathrm{di}}$

$$
G_{r}(-1, t)=\left\langle I\left|\exp \left\{\hat{\mathcal{W}}^{\dagger} t\right\}\right| I\right\rangle\left\langle p^{\mathrm{st}} \mid p^{\mathrm{st}}\right\rangle .
$$

For longer times, even when the system starts to be driven, $|I\rangle$ remains invariant under the time evolution operator as can be seen using Eq. (2) in Eq. (63). We get

$$
G_{r}(-1, T)=M\left\langle p^{\mathrm{st}} \mid p^{\mathrm{st}}\right\rangle \quad \text { for } T \geqslant t_{\mathrm{tr}},
$$

where $M=\langle I \mid I\rangle$ is the total number of states. This implies the following integral FT for the reservoir TEP

$$
M \geqslant\left\langle\exp \left\{-\Delta s_{r}\left[m_{(\tau)}, T\right]\right\}\right\rangle=M \sum_{m=1}^{M}\left(p_{m}^{\mathrm{st}}\left(\lambda_{t_{\mathrm{di}}}\right)\right)^{2} \geqslant 1 .
$$

The equality on the left-hand side [right-hand side] is satisfied if $p_{m}^{\text {st }}\left(\lambda_{t_{\mathrm{di}}}\right)=\delta_{n, m}\left[p_{m}^{\mathrm{st}}\left(\lambda_{t_{\mathrm{di}}}\right)=1 / M\right]$. Jensen's inequality implies $\Delta S_{r}(T, 0) \geqslant 0$. Note that since $\Delta s\left[m_{(\tau)}, T\right]$ is a state function, it is easily verified that

$$
M \sum_{m=1}^{M} p_{m}^{2}(0)=\left\langle\exp \left\{\Delta s\left[m_{(\tau)}, T\right]\right\}\right\rangle
$$

\section{Hatano-Sasa fluctuation theorem}

We assume that the driving starts at the same time or later as the measurement $\left(t_{\mathrm{di}} \geqslant 0\right)$. We define $T^{\prime}>t_{\mathrm{df}}+t_{\mathrm{tr}}$.

We have pointed out at the end of Sec. III that for transitions between steady states $\Delta S_{b}\left(T^{\prime}, 0\right)=0$, so that

$$
\Delta S_{\text {na }}\left(T^{\prime}, 0\right)=\Delta S_{d}\left(T^{\prime}, 0\right)=\Delta S_{d}\left(t_{\mathrm{df}}, t_{\mathrm{di}}\right) \geqslant 0 .
$$

We used the fact that $\Delta S_{d}\left(T^{\prime}, 0\right)$ starts (stops) evolving at $t_{\mathrm{di}}$ $\left(t_{\mathrm{df}}\right)$. The same is true at the trajectory level, since from Eq. (39) we have $\Delta s_{b}\left[m_{(\tau)}, T^{\prime}\right]=0$ and therefore

$$
\Delta s_{\text {na }}\left[m_{(\tau)}, T^{\prime}\right]=\Delta s_{d}\left[m_{(\tau)}, T^{\prime}\right],
$$

where

$$
\begin{aligned}
\Delta s_{d}\left[m_{(\tau)}, T^{\prime}\right] & \equiv \sum_{j=0}^{N} \ln \frac{p_{m_{j}}^{\text {st }}\left(\lambda_{\tau_{j}}\right)}{p_{m_{j}}^{\text {st }}\left(\lambda_{\tau_{j+1}}\right)}=\sum_{j=0}^{N}\left[\phi_{m_{j}}\left(\lambda_{\tau_{j+1}}\right)-\phi_{m_{j}}\left(\lambda_{\tau_{j}}\right)\right] \\
& =\int_{0}^{T^{\prime}} d t \dot{\lambda_{t}} \frac{\partial \phi_{m_{(\tau)}}\left(\lambda_{t}\right)}{\partial \lambda_{t}}=\int_{t_{\mathrm{di}}}^{t_{\mathrm{df}}} d t \dot{\lambda_{t}} \frac{\partial \phi_{m_{(\tau)}}\left(\lambda_{t}\right)}{\partial \lambda_{t}} .
\end{aligned}
$$

The integrand in the third line contributes only during the time intervals between jumps provided the driving is changing. Therefore, if without loss of generality we choose the measurement time such that $T \geqslant t_{\mathrm{df}}$ (if $T<t_{\mathrm{df}}$ one can redefine $t_{\mathrm{df}}$ as equal to $\left.T\right), \Delta s_{d}\left[m_{(\tau)}, T\right]=\Delta s_{d}\left[m_{(\tau)}, T^{\prime}\right]$. This means that for a transition between steady states, the FT (60) reduces to the Hatano-Sasa FT [11]

$$
\left\langle\exp \left\{-s_{d}\left[m_{(\tau)}, T\right]\right\}\right\rangle=1 .
$$

Alternatively, Eq. (72) can be proved from Eq. (58) by showing that when $\gamma=-1, g_{m}^{(d)}(-1, t)=\exp \left\{-\phi_{m}\left(\lambda_{t}\right)\right\}=p_{m}^{\text {st }}\left(\lambda_{t}\right)$ is the solution of Eq. (58).

The FT (72) holds for an arbitrary driving protocol. Let us consider the two extremes. For an adiabatic (infinitely 
slow) driving, the inequality in Eq. (69) becomes an equality. In the other extreme of a sudden driving, where $\lambda_{t}=\lambda_{t_{\mathrm{di}}}$ $+\Theta\left(t-t_{\mathrm{di}}\right)\left(\lambda_{t_{\mathrm{df}}}-\lambda_{t_{\mathrm{di}}}\right)$ and $\dot{\lambda_{t}}=\delta\left(t_{\mathrm{di}}\right)\left(\lambda_{t_{\mathrm{df}}}-\lambda_{t_{\mathrm{di}}}\right)$,

$$
\Delta s_{d}\left[m_{(\tau)}, T\right]=\phi_{m_{0}}\left(\lambda_{t_{\mathrm{df}}}\right)-\phi_{m_{0}}\left(\lambda_{t_{\mathrm{di}}}\right)
$$

becomes a state function and its average takes the simple form

$$
\Delta S_{d}(T, 0)=\sum_{m} p_{m}^{\mathrm{st}}\left(\lambda_{t_{\mathrm{di}}}\right)\left[\phi_{m}\left(\lambda_{t_{\mathrm{df}}}\right)-\phi_{m}\left(\lambda_{t_{\mathrm{di}}}\right)\right] .
$$

Using Eq. (35) with Eq. (70), and since

$$
\Delta s\left[m_{(\tau)}, T\right]=\phi_{m_{T}}\left(\lambda_{t_{\mathrm{df}}}\right)-\phi_{m_{0}}\left(\lambda_{t_{\mathrm{di}}}\right),
$$

we find that

$$
\Delta s_{\mathrm{ex}}\left[m_{(\tau)}, T\right]=\phi_{m_{0}}\left(\lambda_{t_{\mathrm{df}}}\right)-\phi_{m_{T}}\left(\lambda_{t_{\mathrm{df}}}\right)
$$

also becomes a state function and its average becomes

$$
\Delta S_{\mathrm{ex}}(T, 0)=\sum_{m_{0}, m_{T}} p_{m_{0}}^{\mathrm{st}}\left(\lambda_{t_{\mathrm{di}}}\right) p_{m_{T}}^{\mathrm{st}}\left(\lambda_{t_{\mathrm{df}}}\right)\left[\phi_{m_{0}}\left(\lambda_{t_{\mathrm{df}}}\right)-\phi_{m_{T}}\left(\lambda_{t_{\mathrm{df}}}\right)\right] .
$$

\section{Transitions between equilibrium states}

For a system coupled to a single reservoir (or multiple reservoirs with identical thermodynamical properties), the DBC (5) is satisfied. A nondriven system in an arbitrary state will reach after some transient time $t_{\text {tr }}$ the equilibrium grand canonical distribution (6). We again choose $T^{\prime}>t_{\mathrm{df}}+t_{\mathrm{tr}}$ and $T \geqslant t_{\mathrm{df}}$. From the TEP of Sec. IV, we find in this case

$$
\begin{gathered}
\Delta s_{a}\left[m_{(\tau)}, T^{\prime}\right]=0, \quad \Delta s_{r}\left[m_{(\tau)}, T^{\prime}\right]=\Delta s_{\mathrm{ex}}\left[m_{(\tau)}, T^{\prime}\right], \\
\Delta s_{\mathrm{tot}}\left[m_{(\tau)}, T^{\prime}\right]=\Delta s_{\mathrm{na}}\left[m_{(\tau)}, T^{\prime}\right] .
\end{gathered}
$$

The two FTs (59) and (60) become identical and the FT (61) becomes trivial. Using Eq. (6), we also find that Eq. (19) becomes

$$
\phi_{m}(\lambda)=\beta(\lambda)\left\{\epsilon_{m}(\lambda)-\mu(\lambda) N_{m}-\Omega(\lambda)\right\},
$$

where $\Omega(\lambda)=-\ln \Xi(\lambda)$ is the thermodynamic grand canonical potential.

We next consider transitions between equilibrium states, so that the procedure is the same as in Sec. V B 2 but with the DBC (5) now satisfied. We therefore have $\Delta s_{\text {na }}\left[m_{(\tau)}, T^{\prime}\right]=\Delta s_{d}\left[m_{(\tau)}, T^{\prime}\right]$. The driving implies externally modulating the system energies, the chemical potential or the temperature of the reservoir.

When driving the system energy, using Eqs. (71) and (79), we find

$$
\Delta s_{d}\left[m_{(\tau)}, T\right]=\Delta s_{d}\left[m_{(\tau)}, T^{\prime}\right]=\beta w\left[m_{(\tau)}\right]-\beta \Delta \Omega,
$$

where the work is given by $w\left[m_{(\tau)}\right]=\int_{t_{\mathrm{di}}}^{t_{\mathrm{di}}} d t \dot{\epsilon}_{m_{(\tau)}}\left(\lambda_{t}\right)$ and $\Delta \Omega$ $=\Omega\left[\epsilon\left(t_{\mathrm{df}}\right)\right]-\Omega\left[\epsilon\left(t_{\mathrm{di}}\right)\right]$. Both FTs, Eqs. (59) and (60), lead to the same Jarzynski relation [4]

$$
\left\langle\exp \left\{-\beta w\left[m_{(\tau)}\right]\right\}\right\rangle=\exp \{-\beta \Delta \Omega\} .
$$

When driving the reservoir chemical potential, Eqs. (71) and (79) give

$$
\Delta s_{d}\left[m_{(\tau)}, T\right]=\Delta s_{d}\left[m_{(\tau)}, T^{\prime}\right]=\beta \widetilde{w}\left[m_{(\tau)}\right]-\beta \Delta \Omega,
$$

where $\widetilde{w}\left[m_{(\tau)}\right]=-\int_{t_{\mathrm{di}}}^{t_{\mathrm{df}}} d t \dot{\mu} N_{m_{(\tau)}}$ and $\Delta \Omega=\Omega\left[\mu\left(t_{\mathrm{df}}\right)\right]-\Omega\left[\mu\left(t_{\mathrm{di}}\right)\right]$. Both FTs, Eqs. (59) and (60), now lead to

$$
\left\langle\exp \left\{-\beta \widetilde{w}\left[m_{(\tau)}\right]\right\}\right\rangle=\exp \{-\beta \Delta \Omega\} .
$$

The case where reservoir temperature is driven can be calculated similarly.

\section{No driving: Steady state fluctuation theorem}

In a NESS, the relations of Sec. IV give

$$
\begin{gathered}
\Delta s_{\mathrm{na}}\left[m_{(\tau)}, t\right]=\Delta s_{d}\left[m_{(\tau)}, t\right]=0, \\
\Delta s\left[m_{(\tau)}, t\right]=-\Delta s_{\mathrm{ex}}\left[m_{(\tau)}, t\right], \quad \Delta s_{\mathrm{tot}}\left[m_{(\tau)}, t\right]=\Delta s_{a}\left[m_{(\tau)}, t\right] .
\end{gathered}
$$

Furthermore, since $\Delta S(t, 0)=0$, we get

$$
\Delta S_{\text {tot }}(t, 0)=\Delta S_{r}(t, 0) \geqslant 0 .
$$

We shall rewrite the GF evolution equation for the reservoir TEP (53) in the bracket notation

$$
\left|\dot{g}^{(r)}(\gamma, t)\right\rangle=\hat{\mathcal{V}}(\gamma)\left|g^{(r)}(\gamma, t)\right\rangle,
$$

so that

$$
G_{r}(\gamma, t)=\left\langle I|\exp \{\hat{\mathcal{V}}(\gamma) t\}| p^{\mathrm{st}}\right\rangle,
$$

where $|I\rangle$ is a vector with all elements equal to one. Since from (53) the generator has the property $\hat{\mathcal{V}}(\gamma)=\hat{\mathcal{V}}^{\dagger}(-\gamma-1)$, its eigenvalues have the symmetry $s_{\xi}(\gamma)=s_{\xi}(-\gamma-1)$. Furthermore, since $\exp \{\hat{\mathcal{V}}(\gamma) t\}$ is a positive matrix, the FrobeniousPerron theorem [29-31] ensures that all eigenvalues are negative or zero and that the left and the right eigenvectors, $\left|\xi_{0}(\gamma)\right\rangle$ and $\left|\widetilde{\xi}_{0}(\gamma)\right\rangle$, associated with the largest eigenvalue $s_{\xi_{0}}(\gamma)$ exist. Adopting the normalization $\left\langle\tilde{\xi}_{0}(\gamma) \mid \xi_{0}(\gamma)\right\rangle=1$, we find for long times

$$
G_{r}(\gamma, t) \stackrel{t \rightarrow \infty}{=} \exp \left\{s_{\xi_{0}}(\gamma) t\right\}\left\langle I \mid \widetilde{\xi}_{0}(\gamma)\right\rangle\left\langle\xi_{0}(\gamma) \mid p^{\mathrm{st}}\right\rangle,
$$

and that

$$
G_{r}(-\gamma-1, t) \stackrel{t \rightarrow \infty}{=} \exp \left\{s_{\xi_{0}}(\gamma) t\right\}\langle I| \widetilde{\xi}_{0}(-\gamma-1)\left\langle\xi_{0}(-\gamma-1) \mid p^{\mathrm{st}}\right\rangle .
$$

This means that the cumulant generating function

$$
F_{r}(\gamma) \equiv \lim _{t \rightarrow \infty} \frac{1}{t} \ln G_{r}(\gamma, t)
$$

satisfies the symmetry

$$
F_{r}(\gamma)=F_{r}(-\gamma-1) .
$$

Using the theory of large fluctuations this symmetry implies the detailed steady state FT $[6,12]$ 


$$
\frac{P\left(\Delta S_{r}\right)}{P\left(-\Delta S_{r}\right)} \stackrel{t \rightarrow \infty}{=} e^{\Delta S_{r}}
$$

where $P\left(\Delta S_{r}\right)$ is the probability for a trajectory of the system to produce a reservoir TEP equal to $\Delta S_{r}$.

$\Delta s_{r}\left[m_{(\tau)}, t\right]$ grows in average with time because it depends on the number of jumps along the trajectory. However, $\Delta s\left[m_{(\tau)}, t\right]$ is bounded. The FT (92) can therefore be viewed as a consequence of the detailed FT for $\Delta S_{\text {tot }}$ derived in Eq. (A13). The long time limit is needed in order to neglect the contribution from $\Delta s\left[m_{(\tau)}, t\right]$ to $\Delta s_{\text {tot }}\left[m_{(\tau)}, t\right]$.

The FT (67) remains valid at steady state. FTs for currents can also be derived $[19,37,42]$.

\section{ENTROPY FLUCTUATIONS FOR ELECTRON TRANSPORT THROUGH A SINGLE LEVEL QUANTUM DOT}

We have seen in Sec. IV B that the various entropies can be calculated by measuring the different currents between the system and the reservoirs. The counting statistics of electrons through quantum dots has recently raised considerable theoretical [38-42] as well as experimental [27,43-46] interest. The single electrons entering and exiting a quantum dot connected to two leads can be measured. One can therefore calculate all currents, deduce the system trajectories, and calculate the various trajectory entropies presented earlier.

We will analyze the probability distribution for the various trajectory entropies in a single level quantum dot of energy $\epsilon$ connected to two leads with different chemical potentials $\mu_{\nu}(t)$, where $\nu=l, r$. We neglect spin so that the dot can either be empty 0 or filled 1 . The ME is of the form (1) $[40,47-52]$

$$
\left(\begin{array}{l}
\dot{p}_{1}(t) \\
\dot{p}_{0}(t)
\end{array}\right)=\left(\begin{array}{cc}
-v_{t} & w_{t} \\
v_{t} & -w_{t}
\end{array}\right)\left(\begin{array}{l}
p_{1}(t) \\
p_{0}(t)
\end{array}\right)
$$

where

$$
\begin{gathered}
v_{t}=\sum_{\nu} v_{t}^{(\nu)}=\sum_{\nu} a_{\nu}\left[1-f_{\nu}(t)\right], \\
w_{t}=\sum_{\nu} w_{t}^{(\nu)}=\sum_{\nu} a_{\nu} f_{\nu}(t) .
\end{gathered}
$$

The coefficients $a_{\nu}$ characterize the coupling between the dot and the lead $\nu$ with Fermi distribution $f_{\nu}(t) \equiv 1 /(\exp \{\beta\{\epsilon$ $\left.\left.\left.-\mu_{\nu}(t)\right\}\right\}+1\right)$. If $\hbar=1,[a]=[$ energy $]=\left[\right.$ time $\left.^{-1}\right]$. By renormalizing energies by $\epsilon$, all parameters of our model become dimensionless. The steady state distribution of the system is

$$
p_{1}^{(\mathrm{st})}=\frac{w_{t}}{v_{t}+w_{t}}, \quad p_{0}^{(\mathrm{st})}=\frac{v_{t}}{v_{t}+w_{t}},
$$

and the steady state currents are given by

$$
\langle I\rangle_{1,3}^{(\mathrm{st})}=\frac{v_{t}^{(l, r)} w_{t}}{v_{t}+w_{t}}, \quad\langle I\rangle_{2,4}^{(\mathrm{st})}=\frac{w_{t}^{(l, r)} v_{t}}{v_{t}+w_{t}} .
$$

We switch the chemical potential of the left lead $\mu_{l}(t)=\mu_{0}$ $+V(t)$ using the protocol

$$
V(t)=\frac{V_{f}-V_{i}}{2} \tanh \left[c\left(t-t_{m}\right)+1\right]
$$

while holding the right lead chemical potential fixed $\mu_{r}(t)$ $=\mu_{0}$. We can therefore calculate all the trajectory entropies' probability distributions using the GF method described in Sec. IV C. We solve numerically the evolution equations for the $g_{m}(i \gamma, t)$ 's associated with the different entropies for different values of $\gamma$ with the initial condition $g_{m}(i \gamma, 0)$ $=p_{m}(0)$. After calculating the $G(i \gamma, t)$ 's using Eq. (47), the probability distribution is obtained by a numerical inverse Fourier transform Eq. (44). In all calculations we used $\beta$ $=5, \epsilon=1, a_{l}=0.2$, and $a_{r}=0.1$.

We start by analyzing the different contributions to the EP as defined in Sec. III for the $\mu_{l}(t)$ protocol shown in Fig. 2(a).

The system is initially in a nonequilibrium distribution different from the steady state. The solution of the ME (93) as well as its steady state solution are displayed in Fig. 2(b). Between $t=0$ and $t=20, \mu_{l}(t)$ is essentially constant and the system undergoes an exponential relaxation to the steady state. Between $t=20$ and $t=50, \mu_{l}(t)$ changes from $\mu_{0}+V_{i}$ to $\mu_{0}+V_{f}$ fast enough for the system distribution to start differing again from the instantaneous steady state distribution (adiabatic solution). After $t=50, \mu_{l}(t)$ remains constant and the system again undergoes a transient relaxation to the new steady state corresponding to $\mu_{0}+V_{f}$. Figure 2(c) shows the time dependent EP $\dot{S}_{\text {tot }}$ and its adiabatic $\dot{S}_{a}$ and nonadiabatic contribution $\dot{S}_{\text {na. }}$. As predicted, these three quantities are always positive [see Eqs. (21)-(23)]. We also demonstrate that $\dot{S}_{\text {na }}$ only contributes when nonadiabatic effects are significant, i.e., when the actual probability distribution is different from the steady state one $\left[p_{m}(t) \neq p_{m}^{(\mathrm{st})}(\mu(t))\right] . \dot{S}_{a}=0$ only once at $t \approx 33$, when $\mu_{l}(t)=\mu_{r}(t)=0.5$ and the DBC is satisfied. Otherwise the DBC is broken and $\dot{S}_{a}>0$. In Fig. 2(d), we present the two contributions to the nonadiabatic EP $\dot{S}_{\text {na }}$, the driving EP $\dot{S}_{d}$, and the boundary EP $\dot{S}_{b}$ [see Eq. (22)]. The driving EP $\dot{S}_{d}$ only contributes when $\mu_{l}(t)$ changes in time. One can also guess that $\Delta S_{b}=\int_{20}^{60} d t \dot{S}_{b}=0$ due to the fact that the change of boundary EP during an interval between two steady states is zero. $\Delta S_{b}=\int_{0}^{20} d t \dot{S}_{b} \neq 0$ because the system is initially not in a steady state. Figure 2(e) shows the alternative partitioning of the nonadiabatic EP into the system EP $\dot{S}$ and the excess EP $\dot{S}_{\text {ex }}$ [see Eq. (20)]. Finally the splitting of the total EP in the reservoir EP and the system EP [see Eq. (9)] is shown in Fig. 2(f). We see that at steady state $\dot{S}=0$ so that $\dot{S}_{\text {tot }}=\dot{S}_{r}$.

We next study the statistical properties of the different TEP for transitions between steady states. The probability distributions are obtained using the GF method presented in Sec. IV C. The five driving protocols used to change $\mu_{l}(t)$ from $\mu_{0}+V_{i}$ to $\mu_{0}+V_{f}$ are presented in Fig. 3. They range from sudden switch in (i) to slow (adiabatic) switch in (v). The system is always initially in the steady state corresponding to $\mu_{0}+V_{i}$. We will consider measurements which end 

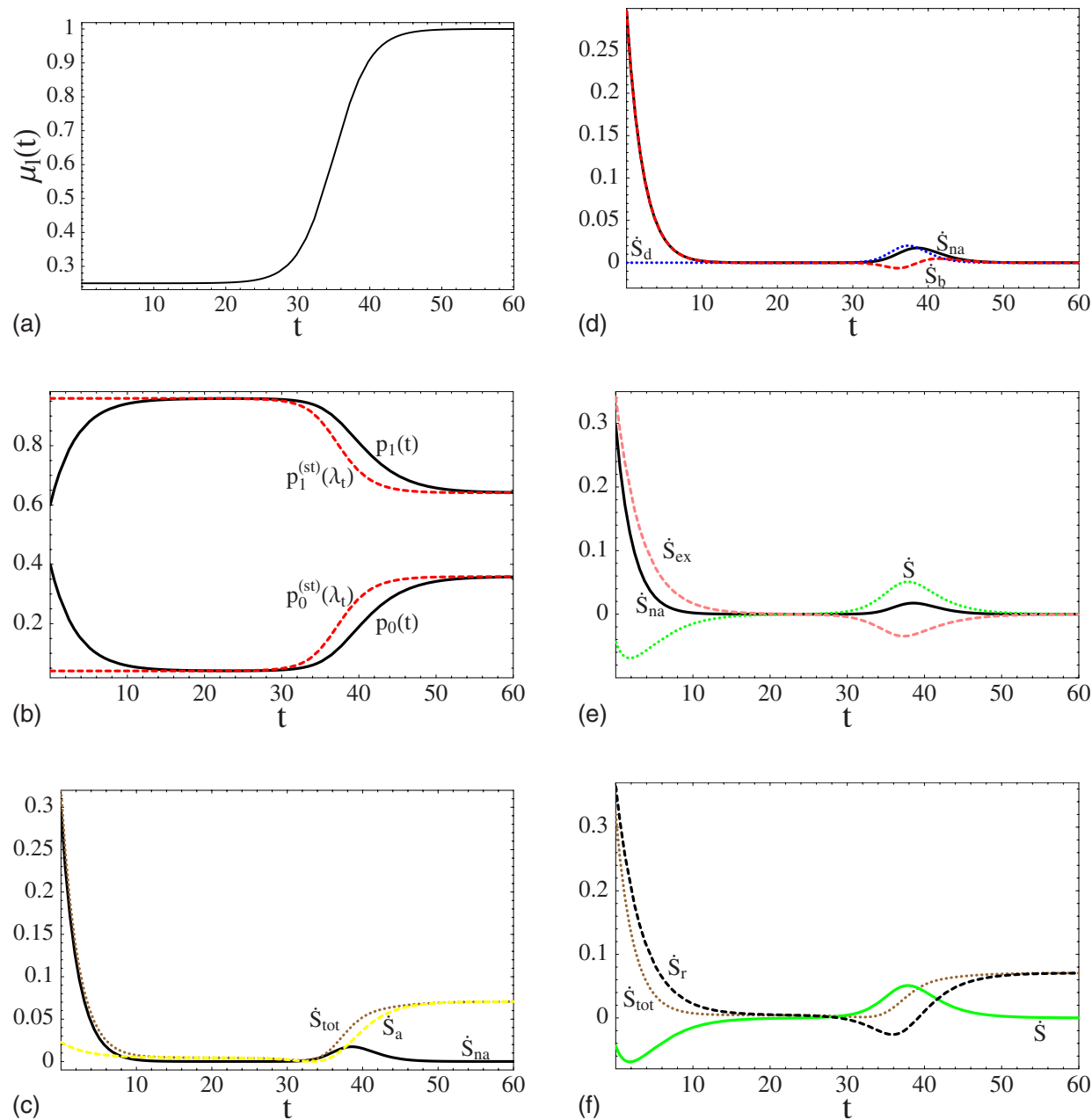

FIG. 2. (Color online) (a) Driving protocol of the left lead chemical potential $\mu_{l}(t)=\mu_{0}$ $+V(t)$ where $V(t)$ follows Eq. (97) with $V_{i}=-0.25, V_{f}=0.5, c=0.2$, $t_{m}=0.2$, and $\mu_{0}=0.5$. The right lead chemical potential remains constant at $\mu_{r}=\mu_{0}$. (b) Solid: The probability distribution of the dot obtained by solving the ME (93) with initial condition $p_{0}(0)=0.4$ and $p_{1}(0)=0.6$. Dotted: The adiabatic probability distribution. (c) Decomposition of $\dot{S}_{\text {tot }}(t)$ according to Eq. (21). (d) Decomposition of $\dot{S}_{\mathrm{na}}(t)$ according to Eq. (22). (e) Decomposition of $\dot{S}_{\mathrm{na}}(t)$ according to Eq. (20). (f) Decomposition of $\dot{S}_{\text {tot }}(t)$ according to Eq. (9). when the system reaches its new steady state at $\mu_{0}+V_{f}$. Different measurement times are represented by $a, b, c, d$.

In Fig. 4, we display $P(\Delta S)$. Since $\Delta s$ is a state function, $P(\Delta S)$ is the same for the various protocol. Because we consider a two level system, $\Delta s$ can only take four possible values which correspond to the four possible changes in the

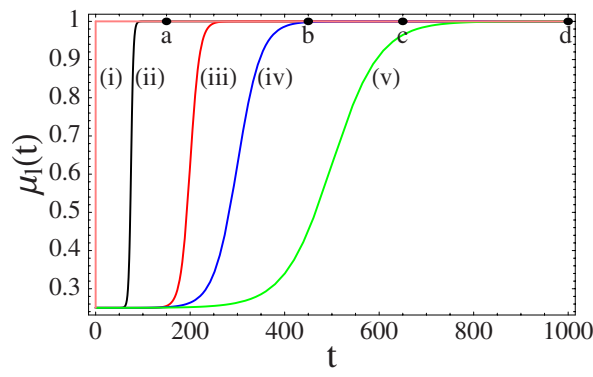

FIG. 3. (Color online) Five driving protocols for the left lead chemical potential $\mu_{l}(t)=\mu_{0}+V(t)$ where $V(t)$ follows Eq. (97) with $V_{i}=-0.25, V_{f}=0.5$ and (ii) $c=0.2, t_{m}=75$, (iii) $c=0.05, t_{m}=200$, (iv) $c=0.02, t_{m}=300$, and (v) $c=0.01, t_{m}=500 . \mu_{r}(t)=\mu_{0}=0.5$. (i) The sudden switch limit $\mu_{l}(t)=\mu_{0}+\Theta(t) V_{f}$ [limit $c \rightarrow \infty$ with $t_{m}=0$ in Eq. (97)]. The system is initially at steady state where $p_{0}^{\text {st }}=0.96$ and $p_{1}^{\text {st }}=0.04$. In the final steady state $p_{0}^{\text {st }}=0.64$ and $p_{1}^{\text {st }}=0.36$. a, b, c, d correspond to different measurement times. In all calculations $\beta$ $=5, \epsilon=1, a_{l}=0.2$, and $a_{r}=0.1$. system state between its initial and final condition. The transitions $0 \rightarrow 0$ and $0 \rightarrow 1$ are much more probable because the probability to initially find the system in the empty state 0 is much higher (0.96) than finding it in the filled state $1(0.04)$. The transition $0 \rightarrow 0$ is more probable than $0 \rightarrow 1$ because the system has a final probability 0.64 to be in its empty state and 0.36 to be in its filled state.

In the left column of Fig. 5, we depict $P\left(\Delta S_{d}\right)$. Here $\Delta s_{b}=0$ so that $\Delta s_{d}=\Delta s_{\text {na }}$ [see Eq. (16)]. All curves (i)-(v) satisfy the FT (60). For the sudden switch (i), $\Delta s_{d}$ becomes a state function which only depends on the initial state of the system [see Eq. (73)]. $\Delta s_{d}$ can therefore take two possible values corresponding to the empty 0 or filled 1 orbital with a



FIG. 4. Probability distribution of the change in the system TEP for the protocols shown in Fig. 3. 

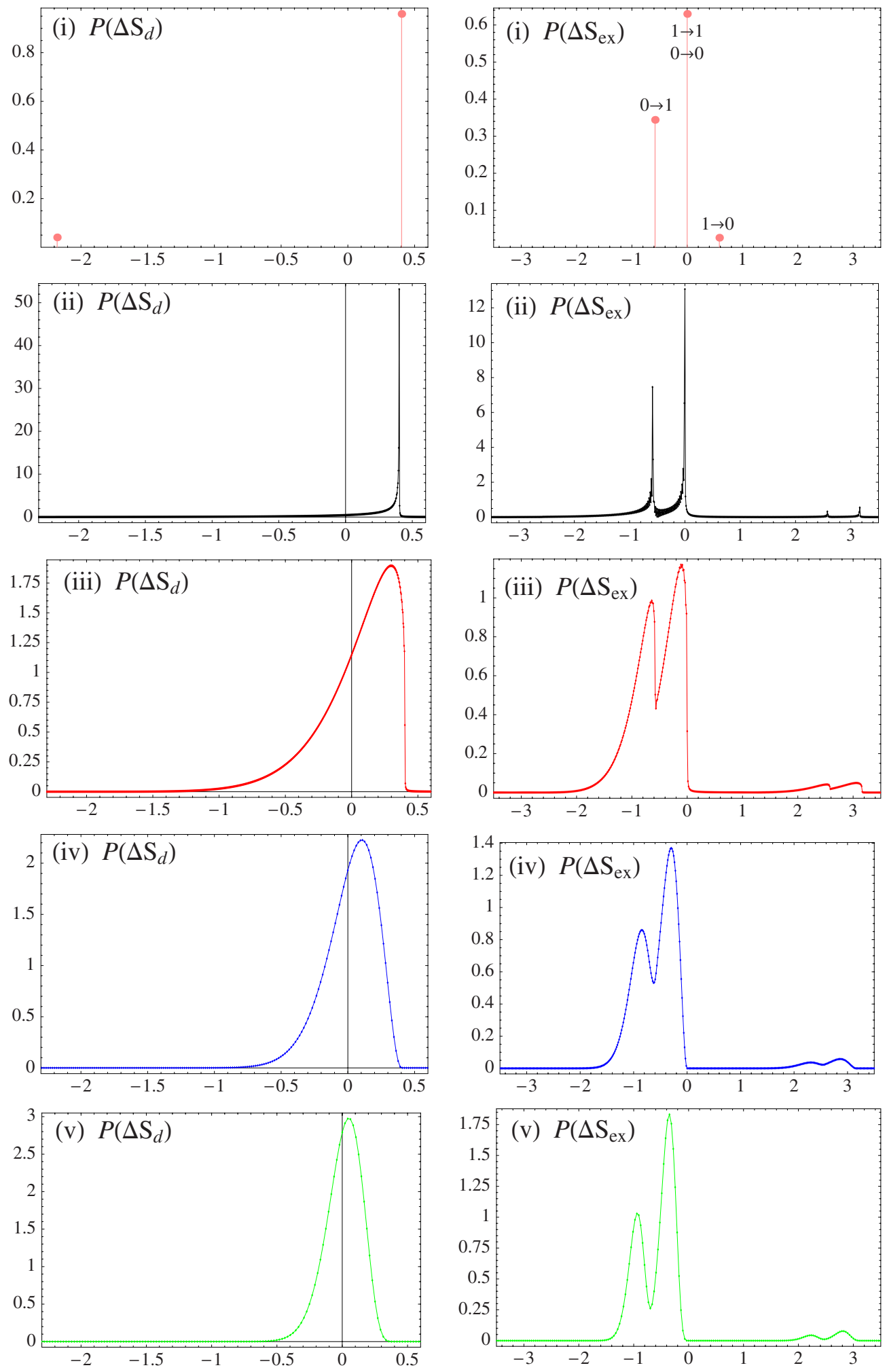

FIG. 5. (Color online) Probability distributions of the change in the excess and driving TEP for the protocols in Fig. 3. All curves in the left column satisfy the FT (60).

respective probability 0.96 or 0.04 . When the driving speed slows down in (ii) the peaks are broadened. In the adiabatic limit $(V), P\left(\Delta S_{d}\right)$ becomes a broad distribution with zero average. In the right column of Fig. 5, we depict $P\left(\Delta S_{\mathrm{ex}}\right)$. For sudden switch (i), $\Delta s_{\mathrm{ex}}$ turns to a state function which only depends on the final steady state distribution [see Eq. (76)]. It is clear from Eq. (76) that the transitions $0 \rightarrow 0$ and $1 \rightarrow 1$ lead to $\Delta s_{\mathrm{ex}}=0$ and $1 \rightarrow 0$ and $0 \rightarrow 1$ to the same $\Delta s_{\mathrm{ex}}$ with opposite sign. The probabilities to observe these transitions follow from the fact that the system is initially more 

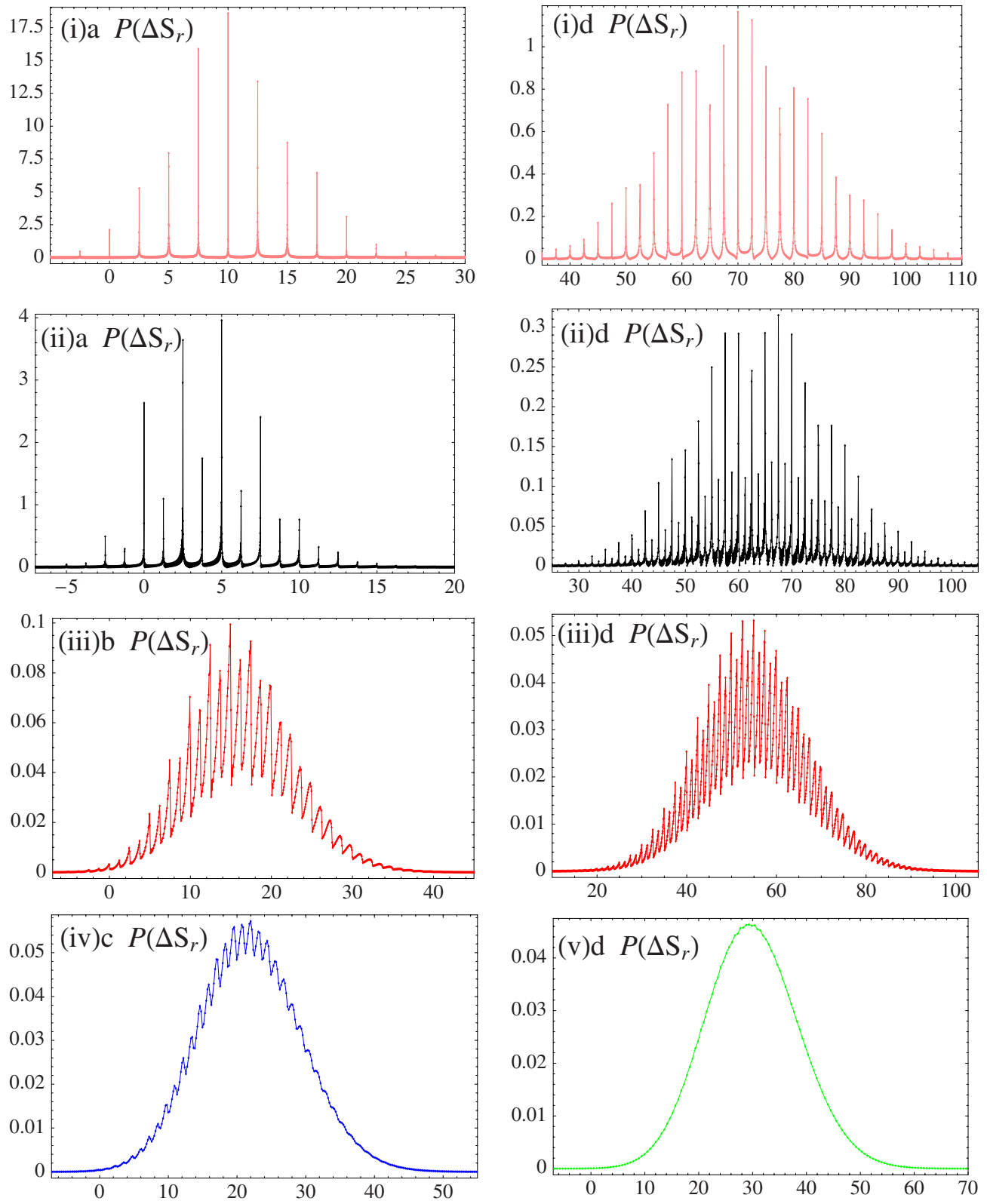

FIG. 6. (Color online) Probability distributions of the change in the reservoir TEP for the driving protocols and measurement times shown in Fig. 3. All curves [except (i)a and (i)d where the driving starts at the same time as the measurement] satisfy the FT (67). likely to be in 0 (prob 0.96) than in 1 . The probability for the final state 0 (1) is $0.64(0.36)$. Therefore, the most likely transition is $0 \rightarrow 0$ followed from $0 \rightarrow 1$. As the driving speed slows down like in (ii), the peaks get broadened. Since $\Delta s_{\mathrm{ex}}=\Delta s_{d}-\Delta s$ [see Eq. (20)] and since in the adiabatic switch limit (v) $P\left(\Delta S_{d}\right)$ is centered around zero, $P\left(\Delta S_{\mathrm{ex}}\right)(\mathrm{v})$ has the same peak structure as $P(-\Delta S)$ (see Fig. 4), but broadened by $\Delta s_{d}$.

In Fig. 6, we display $P\left(\Delta S_{r}\right)$ for different measurement times and protocols. Plots with the same driving but different measurement times [(i)a and (ii)d or (ii)a and (ii)d or (iii)b and (iii)d] show the evolution of $P\left(\Delta S_{r}\right)$ in the final steady state. The plots (ii) $-(\mathrm{v})$ satisfy the FT (67) which for our parameters imply $\left\langle\exp \left\{-\Delta s_{r}\right\}\right\rangle=1.846$. The FT is not satisfied for (i) because the driving starts at the same time as the measurement [see Sec. V B 1]. To understand the structure of $P\left(\Delta S_{r}\right)$, we time integrate Eq. (40) and use the fact that in our model the heat current is proportional to the matter cur- rent between the $\nu$ reservoir and the system $I^{(\nu)}=I_{\text {mat }}^{(\nu)}$ $=I_{\text {heat }}^{(\nu)} / \epsilon$, where

$$
I^{(\nu)}(t)=\sum_{j=1}^{N} \delta_{\nu, \nu_{j}}\left(t-\tau_{j}\right)\left(N_{m_{j}}-N_{m_{j-1}}\right) .
$$

We get

$$
\Delta s_{r}(t)=-\beta \sum_{\nu} \int_{0}^{t} d \tau\left(\epsilon-\mu_{\nu}(\tau)\right) I^{(\nu)}(\tau) .
$$

In the sudden switch limit (i), we get

$$
\Delta s_{r}(t)=-\beta \sum_{\nu}\left(\epsilon-\mu_{\nu}(T)\right) \mathcal{N}^{(\nu)}(t),
$$

where $\mathcal{N}^{(\nu)}=\int_{0}^{t} d \tau I^{(\nu)}(\tau)$ is the net number of electron transferred from the reservoir $\nu$ to the system between 0 and $t$. This explains why $\Delta s_{r}$ in (i) only takes a discrete value 

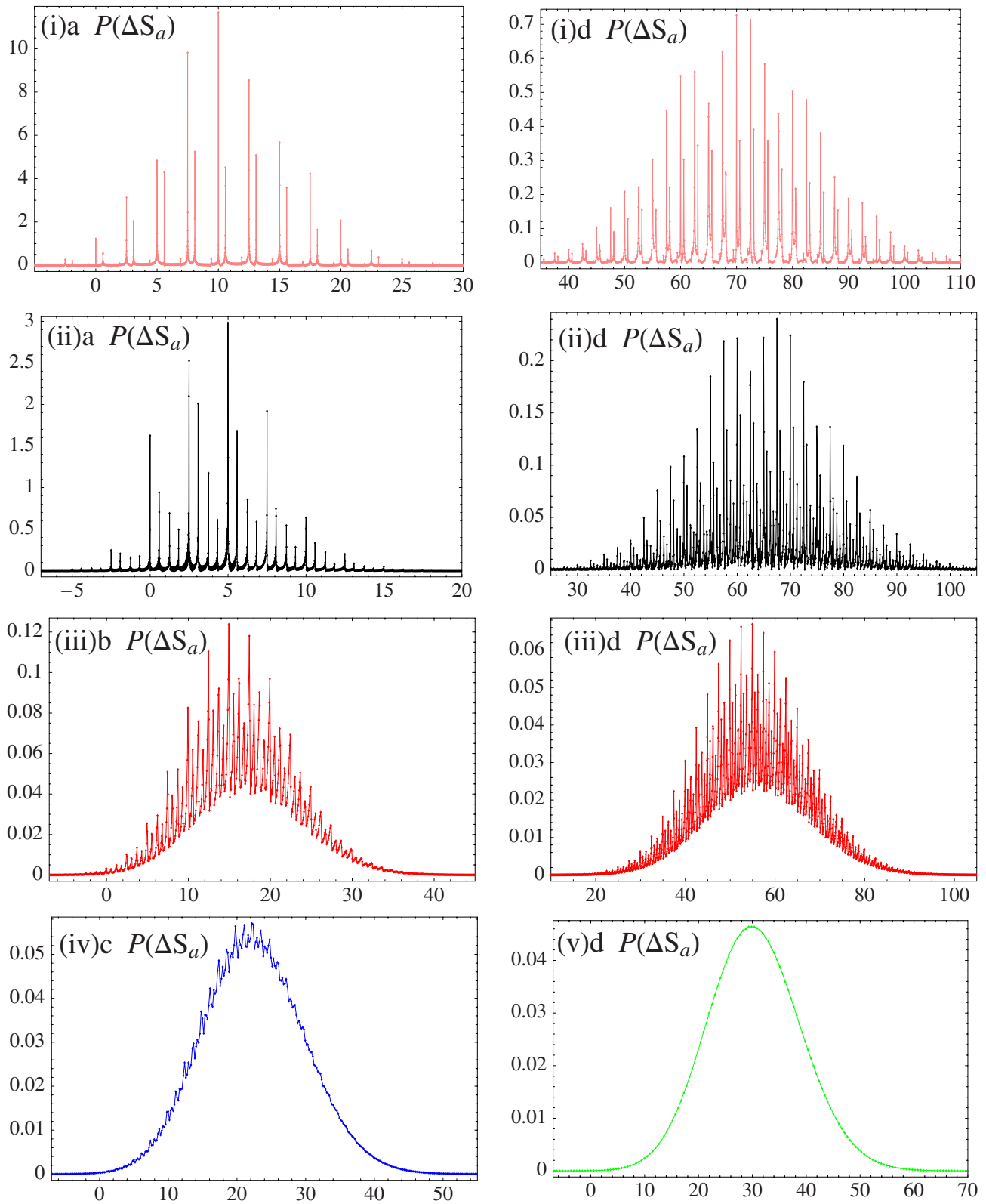

In Fig. 8, we show $P\left(\Delta S_{\text {tot }}\right)$. All curves satisfy the FT (59) [verification was done on the GF (not shown)]. The structure of $P\left(\Delta S_{\text {tot }}\right)$ can be understood using $P\left(\Delta S_{r}\right)$ and $P(\Delta S)$ because $\Delta s_{\text {tot }}=\Delta s_{r}+\Delta s$. This is clear for the sudden switch (i) where the peaks of $P\left(\Delta S_{r}\right)$ are split in smaller peaks which have the structure of $P(\Delta S)$.

\section{CONCLUSIONS}

For a driven open system in contact with multiple reservoirs and described by a master equation, we have proposed a partitioning of the trajectory entropy production into two parts. One contributes when the system is not in its steady state and contains two contributions due to the external driving and the deviation from steady state in the initial and final probability distribution of the system. The second part comes from breaking of the detailed balance condition by the multiple reservoirs and becomes equal to the total entropy pro- 

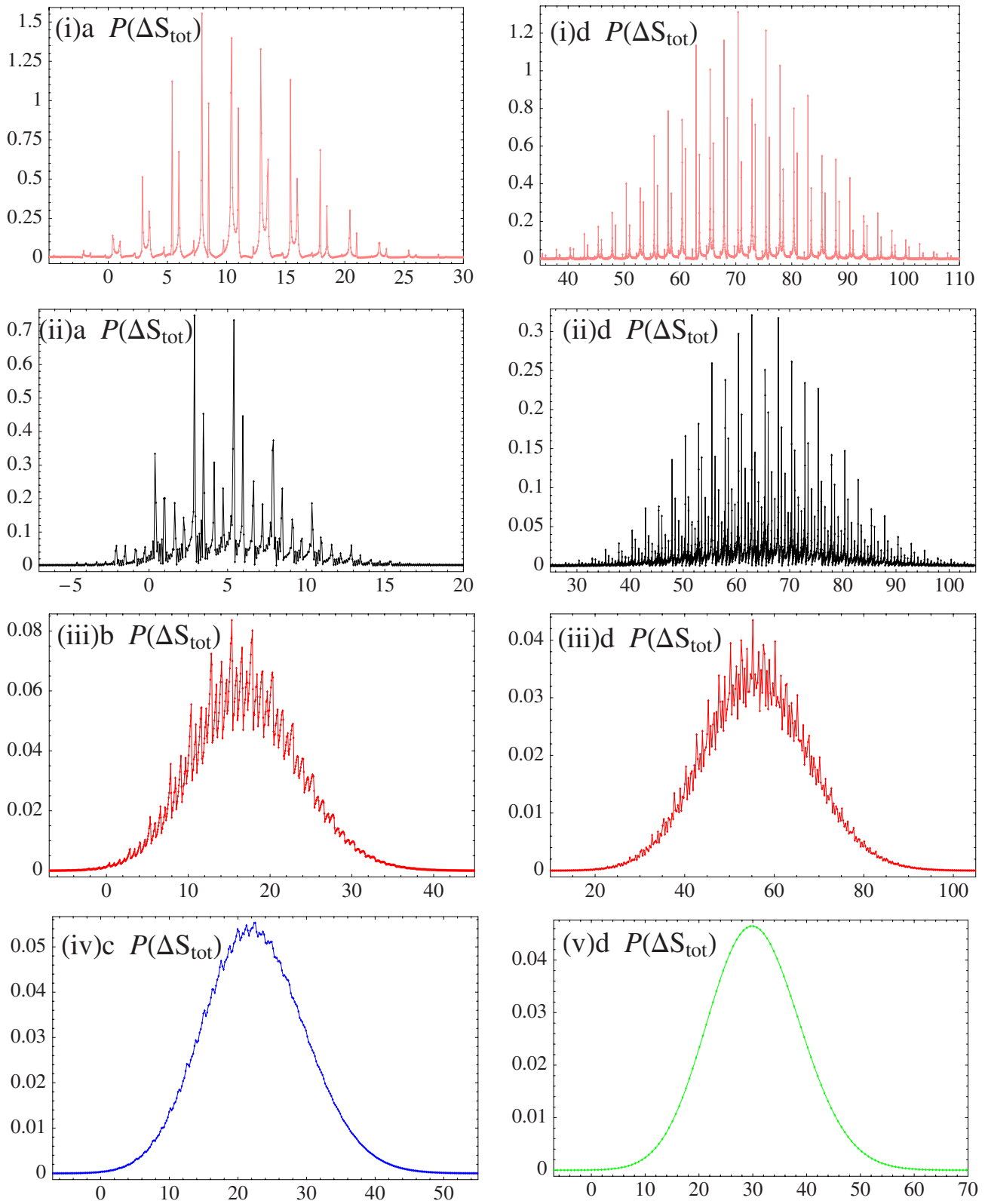

FIG. 8. (Color online) Probability distributions of the change in the total TEP for the driving protocols and measurement times shown in Fig. 3. All curves satisfy the FT (59). duction when the system remains in its steady state all throughout the nonequilibrium process. Both parts as well as the total entropy production satisfy a general integral fluctuation theorem which imposes positivity on their ensemble average. This partitioning also provides a simple way to identify which part of the entropy production contributes during a specific type of nonequilibrium process (see Fig. 9). Previously derived integral fluctuation theorems can be recovered from our three general fluctuation theorems and in addition we derived a new integral fluctuation theorem for the part of the entropy production due to exchange processes between the system and its reservoirs (reservoir entropy production). Our results strictly apply to systems described by a master equation (1). However, as has often been the case for previous fluctuation relations, one could expect similar results to hold for other types of dynamics. For electron transport through a single level quantum dot between two reservoirs with time dependent chemical potentials, we have simulated and analyzed in detail the probability distributions of the various trajectory entropies and showed how they can be measured in electron counting statistics experiments.

\section{ACKNOWLEDGMENTS}

The support of the National Science Foundation (Grant No. CHE-0446555) and NIRT (Grant No. EEC 0303389) is gratefully acknowledged. M.E. is partially supported by the FNRS Belgium (collaborateur scientifique).

\section{APPENDIX: FLUCTUATION THEOREMS IN TERMS OF FORWARD BACKWARD TRAJECTORY PROBABILITIES}

We show that the FTs (59) and (60) have an interesting interpretation in terms of the ratio of the probability of a forward dynamics generating a given trajectory and the probability of the time-reversed trajectory during some backward dynamics. This is an alternative to the GF approach which 


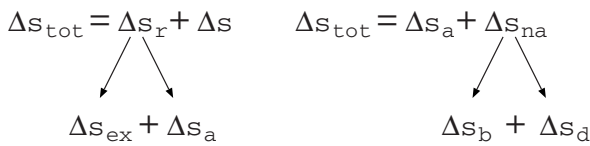

\begin{tabular}{c|c|c|c|} 
& $\Delta \mathbf{s}_{\mathrm{a}}$ & $\Delta \mathbf{s}_{\mathrm{b}}$ & $\Delta \mathbf{s}_{\mathrm{d}}$ \\
\hline SS-SS & & 0 & \\
Eq-Eq & 0 & 0 & \\
AdTrf & & 0 & $<>=0$ \\
TRSS & & & 0 \\
TREq & 0 & & 0 \\
SS & & 0 & 0 \\
Eq & 0 & 0 & 0
\end{tabular}

FIG. 9. Summary of the two splittings of the total TEP in different parts and of the type of transformations during which these parts are zero. SS-SS: Transition between steady states. Eq-Eq: Transition between equilibrium states. AdTrf: Adiabatic transformation. TRSS: Transient relaxation to steady state. TREq: Transient relaxation to equilibrium. SS: steady state. Eq: Equilibrium. \langle\rangle$=0$ means that the ensemble average vanishes.

connects the detailed form to the integral form of the FTs.

The forward dynamics is described by the ME (1). We introduce the probability (in trajectory space) $\mathcal{P}\left[m_{(\tau)}\right]$ that the system follows a trajectory $m_{(\tau)}$

$$
\begin{aligned}
\mathcal{P}\left[m_{(\tau)}\right]= & p_{m_{0}}(0)\left[\prod_{j=1}^{N} \exp \left(\int_{\tau_{j-1}}^{\tau_{j}} d \tau^{\prime} W_{m_{j-1}, m_{j-1}}\left(\lambda_{\tau^{\prime}}\right)\right)\right. \\
& \left.\times W_{m_{j}, m_{j-1}}^{\left(\nu_{j}\right)}\left(\lambda_{\tau_{j}}\right)\right] \exp \left(\int_{\tau_{N}}^{T} d \tau^{\prime} W_{m_{N}, m_{N}}\left(\lambda_{\tau^{\prime}}\right)\right) .
\end{aligned}
$$

The $W_{m_{i}, m_{j-1}}^{\left(\nu_{j}\right)}\left(\lambda_{\tau_{j}}\right)$ factors in this expression represent the probability that the system undergoes a given transition whereas the exponentials describe the probability for the system to remain in a given state between two successive jumps. Summation over all possible trajectories will be denoted by $\Sigma_{m_{(\tau)}}$. It consists of time-ordered integrations over the $N$ time variables $\tau_{j}$ from 0 to $T$ (this gives the probability of having a path with $N$ transitions) and then summing over all possible $N$ from 0 to $\infty$. Normalization in trajectory-space implies that $\sum_{m_{(\tau)}} \mathcal{P}\left[m_{(\tau)}\right]=1$.

The backward dynamics is described on the time interval $t=[0, T]$ by the ME

$$
\dot{\tilde{p}}_{m}(t)=\sum_{m^{\prime}} \widetilde{W}_{m, m^{\prime}}\left(\lambda_{T-t}\right) \widetilde{p}_{m^{\prime}}(t),
$$

where the new rate matrix $\widetilde{W}_{m, m^{\prime}}\left(\lambda_{t}\right)$ satisfies $\Sigma_{m} \widetilde{W}_{m, m^{\prime}}\left(\lambda_{t}\right)$ $=0$. We require that the parametric time dependence (via the driving protocol $\lambda_{t}$ ) of the rate matrix in Eq. (A2) is timereversed compared that of Eq. (1) and that the diagonal part of the rate matrix in Eqs. (1) and (A2) is the same

$$
\widetilde{W}_{m, m}\left(\lambda_{t}\right)=W_{m, m}\left(\lambda_{t}\right) .
$$

This still leaves room for different choices of $\widetilde{W}_{m, m^{\prime}}\left(\lambda_{t}\right)$. We will later specify two choice of $\widetilde{W}_{m, m^{\prime}}\left(\lambda_{T-t}\right)$ [Eqs. (A11) and (A14)] that will result in two FTs.

We define the time-reversed trajectory of $m_{(\tau)}$ by

$$
\begin{aligned}
\bar{m}_{(\tau)}= & \left\{0-m_{N} \stackrel{T-\tau_{N}}{\longrightarrow} m_{N-1} \stackrel{T-\tau_{N-1}}{\longrightarrow} \cdots m_{j} \stackrel{T-\tau_{j}}{\longrightarrow} m_{j-1}\right. \\
& \left.\stackrel{T-\tau_{j-1}}{\longrightarrow} \cdots m_{1} \stackrel{T-\tau_{1}}{\longrightarrow} m_{0}-T\right\} .
\end{aligned}
$$

The probability $\tilde{\mathcal{P}}\left[\bar{m}_{(\tau)}\right]$ that the system described by Eq. (A2) follows the time-reversed trajectory $\bar{m}_{(\tau)}$ is given by

$$
\begin{aligned}
\tilde{\mathcal{P}}\left[\bar{m}_{(\tau)}\right]= & \tilde{p}_{m_{N}}(0)\left[\prod _ { j = 1 } ^ { N } \operatorname { e x p } \left(\int_{T-\tau_{N-j+2}}^{T-\tau_{N-j+1}} d \tau^{\prime}\right.\right. \\
& \left.\times \widetilde{W}_{m_{N-j+1}, m_{N-j+1}}\left(\lambda_{T-\tau^{\prime}}\right)\right) \\
& \times \widetilde{W}_{m_{N-j}\left(\nu_{N-j+1}\right)}\left(\lambda_{N-j+1}\right. \\
& \times \exp \left(\int_{T-\tau_{N-j+1}}^{T} d \tau^{\prime} \tilde{W}_{m_{0}, m_{0}}\left(\lambda_{T-\tau^{\prime}}\right)\right),
\end{aligned}
$$

where $\tau_{N+1}=T$. Normalization in the reverse path ensemble implies $\Sigma_{\bar{m}_{(\tau)}} \tilde{\mathcal{P}}\left[\bar{m}_{(\tau)}\right]=1$.

We consider the ratio of the two probabilities (A1) and (A4),

$$
r\left[m_{(\tau)}\right] \equiv \ln \frac{\mathcal{P}\left[m_{(\tau)}\right]}{\widetilde{\mathcal{P}}\left[\bar{m}_{(\tau)}\right]} .
$$

Due to Eq. (A3), the contributions from the exponentials (which represent the probabilities to remain on a given state) in Eq. (A5) cancel, so that

$$
r\left[m_{(\tau)}\right]=\ln \frac{p_{m_{0}}(0)}{\widetilde{p}_{m_{N}}(0)}+\sum_{j=1}^{N} \ln \frac{W_{m_{j}, m_{j-1}}^{\left(\nu_{j}\right)}\left(\lambda_{\tau_{j}}\right)}{\widetilde{W}_{m_{j-1}, m_{j}}^{\left(\nu_{j}\right)}\left(\lambda_{\tau_{j}}\right)} .
$$

We can partition Eq. (A6) in the form

$$
\begin{aligned}
r\left[m_{(\tau)}\right]= & \ln \frac{p_{m_{0}}(0)}{\widetilde{p}_{m_{N}}(0)}+\sum_{j=1}^{N} \ln \frac{p_{m_{j}}^{\mathrm{st}}\left(\lambda_{\tau_{j}}\right)}{p_{m_{j-1}}^{\mathrm{st}}\left(\lambda_{\tau_{j}}\right)} \\
& +\left(\sum_{j=1}^{N} \ln \frac{p_{m_{j-1}}^{\mathrm{st}}\left(\lambda_{\tau_{j}}\right) W_{m_{j}, m_{j-1}}^{\left(\nu_{j}\right)}\left(\lambda_{\tau_{j}}\right)}{p_{m_{j}}^{\mathrm{st}}\left(\lambda_{\tau_{j}}\right) \tilde{W}_{m_{j-1}, m_{j}}^{\left(\nu_{j}\right)}\left(\lambda_{\tau_{j}}\right)}\right) .
\end{aligned}
$$

We assume for the moment that $r\left[m_{(\tau)}\right]$ can be expressed exclusively in terms of quantities of the dynamics (1), i.e., a recipe has to be provided to express the tilde quantities in 
Eq. (A6) $\left[\tilde{p}_{m_{N}}(0)\right.$ and $\left.\tilde{W}_{m_{j-1}, m_{j}}^{\left(\nu_{j}\right)}\left(\lambda_{\tau_{j}}\right)\right]$ in terms of nontilde quantities.

In analogy with Eq. (A5), we define

$$
\widetilde{r}\left[\bar{m}_{(\tau)}\right] \equiv \ln \frac{\tilde{\mathcal{P}}\left[\bar{m}_{(\tau)}\right]}{\mathcal{P}\left[m_{(\tau)}\right]}
$$

for the tilde dynamics. The previous recipe also implies that $\widetilde{r}\left[\bar{m}_{(\tau)}\right]$ can be exclusively expressed in terms of quantities of the tilde dynamics (A2). Equation (A5) together with Eq. (A8) implies that $r\left[m_{(\tau)}\right]=-\widetilde{r}\left[\bar{m}_{(\tau)}\right]$.

The probability $P(R)$ to observe a trajectory such that $r\left[m_{(\tau)}\right]=R$ during the forward dynamics is related to the probability $\widetilde{P}(-R)$ to observe a trajectory such that $\widetilde{r}\left[\bar{m}_{(\tau)}\right]$ $=-R$ during the backward dynamics

$$
\begin{aligned}
P(R) & \equiv \sum_{m_{(\tau)}} \mathcal{P}\left[m_{(\tau)}\right] \delta\left(R-r\left[m_{(\tau)}\right]\right) \\
& =\sum_{m_{(\tau)}} \tilde{\mathcal{P}}\left[\bar{m}_{(\tau)}\right] e^{r\left[m_{(\tau)}\right]} \delta\left(R-r\left[m_{(\tau)}\right]\right) \\
& =e^{R} \sum_{m_{(\tau)}} \tilde{\mathcal{P}}\left[\bar{m}_{(\tau)}\right] \delta\left(R-r\left[m_{(\tau)}\right]\right) \\
& =e^{R} \sum_{\bar{m}_{(\tau)}} \tilde{\mathcal{P}}\left[\bar{m}_{(\tau)}\right] \delta\left(R+\widetilde{r}\left[\bar{m}_{(\tau)}\right]\right)=e^{R} \widetilde{P}(-R) .
\end{aligned}
$$

By integrating $e^{-R} P(R)=\widetilde{P}(-R)$ over $R$, we get

$$
\left\langle e^{-r\left[m_{(\tau)}\right]}\right\rangle=1 .
$$

It follows from Jensen's inequality $\left\langle e^{x}\right\rangle \geqslant e^{\langle x\rangle}$, that $\left\langle r\left[m_{(\tau)}\right]\right\rangle$ $\geqslant 0$.

We now make a first choice of $\tilde{W}_{m, m^{\prime}}\left(\lambda_{t}\right)$ in the backward dynamics (A2)

$$
\tilde{W}_{m, m^{\prime}}^{(\nu)}\left(\lambda_{t}\right)=W_{m, m^{\prime}}^{(\nu)}\left(\lambda_{t}\right)
$$

In this case the backward dynamics is identical to the original one, except that the driving protocol is time reversed. If we also choose the initial conditions of the backward dynamics to be the final conditions of the forward dynamics $\tilde{p}_{m}(0)=p_{m}(T)$, using Eqs. (A6) and (27), we find

$$
r\left[m_{(\tau)}\right]=\Delta s_{\text {tot }}\left[m_{(\tau)}, T\right] .
$$

The FT (59) previously derived using GFs now follows from Eq. (A10). Using Eq. (A9), we also get the detailed form of the FT

$$
\frac{P\left(\Delta S_{\mathrm{tot}}\right)}{\widetilde{P}\left(-\Delta S_{\mathrm{tot}}\right)}=e^{\Delta S_{\mathrm{tot}}} .
$$

We now make a second choice of $\widetilde{W}_{m, m^{\prime}}\left(\lambda_{t}\right)$ in Eq. (A2)

$$
\tilde{W}_{m, m^{\prime}}^{(\nu)}\left(\lambda_{t}\right)=W_{m^{\prime}, m}^{(\nu)}\left(\lambda_{t}\right) \frac{p_{m}^{\mathrm{st}}\left(\lambda_{t}\right)}{p_{m^{\prime}}^{\mathrm{st}}\left(\lambda_{t}\right)} .
$$

In the theory of MEs, Eq. (A2) with Eq. (A14) is called the time reversal ME of Eq. (1) [29,30]. We again choose the initial condition of the tilde dynamics to be the final conditions of the original dynamics $\tilde{p}_{m}(0)=p_{m}(T)$. Using Eq. (A7) with Eqs. (A14) and (32), we get

$$
r\left[m_{(\tau)}\right]=\Delta s_{\mathrm{na}}\left[m_{(\tau)}, T\right] .
$$

The previously derived FT (60) follows now from Eq. (A10). From Eq. (A9) we find the detailed form of the FT

$$
\frac{P\left(\Delta S_{\mathrm{na}}\right)}{\widetilde{P}\left(-\Delta S_{\mathrm{na}}\right)}=e^{\Delta S_{\mathrm{na}}} .
$$

We can interpret the change in the total TEP during the 0 to $T$ time interval as the logarithm of the (forward) probability that the driven system follows a given trajectory divided the backward probability that the system, initially in the final probability distribution of the forward evolution, and driven in a time reversed way compared to the forward evolution, follows the time-reversed trajectory.

The nonadiabatic TEP is interpreted as the logarithm of the (forward) probability that the driven system follows a given trajectory divided by the backward probability that the system, initially in the final probability distribution of the forward evolution, and described by the time-reversed $\mathrm{ME}$, follows the time-reversed trajectory.

It should be noted that the backward ME (A2) with (A14) is different from the backward ME (A2) with (A11) only for systems interacting with multiple reservoirs which break the DBC. Only in this case Eq. (59) is different from Eq. (60).
[1] D. J. Evans, E. G. D. Cohen, and G. P. Morriss, Phys. Rev. Lett. 71, 2401 (1993).

[2] G. Gallavotti and E. G. D. Cohen, Phys. Rev. Lett. 74, 2694 (1995); J. Stat. Phys. 80, 931 (1995).

[3] D. J. Evans and D. J. Searles, Phys. Rev. E 50, 1645 (1994); 52, 5839 (1995); 53, 5808 (1996).

[4] C. Jarzynski, Phys. Rev. Lett. 78, 2690 (1997); Phys. Rev. E 56, 5018 (1997).

[5] J. Kurchan, J. Phys. A 31, 3719 (1998).

[6] J. L. Lebowitz and H. Spohn, J. Stat. Phys. 95, 333 (1999).

[7] D. J. Searles and D. J. Evans, Phys. Rev. E 60, 159 (1999).
[8] G. E. Crooks, Phys. Rev. E 60, 2721 (1999).

[9] G. E. Crooks, Phys. Rev. E 61, 2361 (2000).

[10] T. Hatano, Phys. Rev. E 60, R5017 (1999).

[11] T. Hatano and S. I. Sasa, Phys. Rev. Lett. 86, 3463 (2001).

[12] P. Gaspard, J. Chem. Phys. 120, 8898 (2004).

[13] D. Andrieux and P. Gaspard, J. Chem. Phys. 121, 6167 (2004).

[14] U. Seifert, Phys. Rev. Lett. 95, 040602 (2005).

[15] T. Speck and U. Seifert, J. Phys. A 38, L581 (2005).

[16] V. Y. Chernyak, M. Chertkov, and C. Jarzynski, J. Stat. Mech.: Theory Exp. 2006, P08001 (2006).

[17] B. Cleuren, C. Van den Broeck, and R. Kawai, Phys. Rev. E 
74, 021117 (2006).

[18] C. Bustamante, J. Liphardt, and F. Ritort, Phys. Today 58, 43 (2005).

[19] D. Andrieux and P. Gaspard, J. Stat. Mech.: Theory Exp. 2006, P01011 (2006).

[20] J. Liphardt, S. Dumont, S. B. Smith, I. Tinoco, Jr., and C. Bustamante, Science 296, 1832 (2002).

[21] E. H. Trepagnier, C. Jarzynski, F. Ritort, G. E. Crooks, C. J. Bustamante, and J. Liphardt, Proc. Natl. Acad. Sci. U.S.A. 101, 15038 (2004).

[22] D. Collin, F. Ritort, C. Jarzynski, S. B. Smith, I. Tinoco, Jr., and C. Bustamante, Nature (London) 437, 231 (2005).

[23] F. Douarche, S. Ciliberto, A. Petrosyan, and I. Rabbiosi, Europhys. Lett. 70, 593 (2005).

[24] G. M. Wang, J. C. Reid, D. M. Carberry, D. R. M. Williams, E. M. Sevick, and D. J. Evans, Phys. Rev. E 71, 046142 (2005).

[25] S. Schuler, T. Speck, C. Tietz, J. Wrachtrup, and U. Seifert, Phys. Rev. Lett. 94, 180602 (2005).

[26] C. Tietz, S. Schuler, T. Speck, U. Seifert, and J. Wrachtrup, Phys. Rev. Lett. 97, 050602 (2006).

[27] T. Fujisawa, T. Hayashi, R. Tomita, and Y. Hirayama, Science 312, 1634 (2006).

[28] We assume an irreducible rate matrix [29-31].

[29] D. Stirzaker, Stochastic Processes and Models (Oxford University Press, New York, 2005).

[30] J. R. Norris, Markov Chains (Cambridge University Press, Cambridge, UK, 1997).

[31] N. G. van Kampen, Stochastic Processes in Physics and Chemistry, 2nd ed. (North-Holland, Amsterdam, 1997).

[32] D. Kondepudi and I. Prigogine, Modern Thermodynamics (Wiley, Chichester, 1998).

[33] S. R. de Groot and P. Mazur, Non-Equilibrium Thermodynamics (Dover, New York, 1984).

[34] J. Schnakenberg, Rev. Mod. Phys. 48, 571 (1976).
[35] Y. Oono and M. Paniconi, Prog. Theor. Phys. Suppl. 130, 29 (1998).

[36] S. I. Sasa and H. Tasaki, arXiv:cond-mat/0411052.

[37] D. Andrieux and P. Gaspard, J. Stat. Phys. 127, 107 (2007).

[38] L. S. Levitov and M. Reznikov, Phys. Rev. B 70, 115305 (2004).

[39] A. L. Shelankov and J. Rammer, Europhys. Lett. 63, 485 (2003).

[40] D. A. Bagrets and Yu. V. Nazarov, Phys. Rev. B 67, 085316 (2003).

[41] Y. Utsumi, D. S. Golubev, and G. Schön, Phys. Rev. Lett. 96, 086803 (2006)

[42] M. Esposito, U. Harbola, and S. Mukamel, Phys. Rev. B 75, 155316 (2007).

[43] W. Lu, Z. Ji, L. Pfeiffer, K. W. West, and A. J. Rimberg, Nature (London) 423, 422 (2003).

[44] T. Fujisawa, T. Hayashi, Y. Hirayama, and H. D. Cheong, Appl. Phys. Lett. 84, 2343 (2004).

[45] J. Bylander, T. Duty, and P. Delsing, Nature (London) 434, 361 (2005).

[46] S. Gustavsson, R. Leturcq, B. Simovic, R. Schleser, T. Ihn, P. Studerus, K. Ensslin, D. C. Driscoll, and A. C. Gossard, Phys. Rev. Lett. 96, 076605 (2006).

[47] C. W. J. Beenakker, Phys. Rev. B 44, 1646 (1991).

[48] E. Bonet, M. M. Deshmukh, and D. C. Ralph, Phys. Rev. B 65, 045317 (2002).

[49] F. Elste and C. Timm, Phys. Rev. B 71, 155403 (2005).

[50] S. Datta, Quantum Transport: From Quantum Dot to Transistor (Cambridge, New York, 2005).

[51] B. Muralidharan, A. W. Ghosh, and S. Datta, Phys. Rev. B 73, 155410 (2006).

[52] U. Harbola, M. Esposito, and S. Mukamel, Phys. Rev. B 74, 235309 (2006) 